\title{
Convergence of Shock Capturing Schemes for the Compressible Euler-Poisson Equations
}

\author{
G.-Q. Chen ${ }^{1,2}$, D. Wang ${ }^{2}$ \\ ${ }^{1}$ Department of Mathematics, Northwestern University, 2033 Sheridan Road, Evanston, IL 60208, \\ USA Email: gqchen@math.nwu.edu \\ 2 Department of Mathematics, University of Chicago, 5734 S University Avenue, Chicago, \\ IL 60637, USA. Email: dwang@math.uchicago edu
}

Received: 4 April 1995/Accepted: 14 November 1995

\begin{abstract}
We are concerned with approximate methods to construct global solutions with geometrical structure to the compressible Euler-Poisson equations in several space variables. A shock capturing numerical scheme is introduced to overcome the new difficulties from the nonlinear resonance of the system and the nonlocal behavior of the source terms. The convergence and consistency of the shock capturing scheme for the equations is proved with the aid of the compensated compactness method. Then new existence results of the global solutions with geometrical structure are obtained. The traces of the weak solutions are defined and then the weak solutions are proved to satisfy the boundary conditions. The initial data are arbitrarily large with $L^{\infty}$ bounds.
\end{abstract}

\section{Introduction}

We consider the Euler-Poisson equations for compressible flows with the form

$$
\left\{\begin{array}{l}
\rho_{t}+\nabla \cdot \vec{m}=0, \\
\vec{m}_{t}+\nabla \cdot\left(\frac{\vec{m} \otimes \vec{m}}{\rho}\right)+\nabla p(\rho)=\rho \nabla \phi-\frac{\vec{m}}{\tau}, \\
\Delta \phi=\rho-D(\vec{x}),
\end{array}\right.
$$

where $\rho(\vec{x}, t) \in \mathbf{R}, \vec{m}(\vec{x}, t) \in \mathbf{R}^{N}$, and $\phi(\vec{x}, t) \in \mathbf{R}$ denote the density, the mass, and the potential of the flows, respectively; $p(\rho)=\rho^{\gamma} / \gamma, \gamma \in\left(1, \frac{5}{3}\right]$, is the pressuredensity relation function; $\tau>0$ is the momentum relaxation time; $D(\vec{x})$ is the doping profile; and $\Delta \phi=\frac{\partial^{2} \phi}{\partial x_{1}^{2}}+\cdots+\frac{\partial^{2} \phi}{\partial x_{N}^{2}}$ is the Laplacian of $\phi$ in $\mathbf{R}^{N}$. On the non-vacuum states $(\rho>0), \vec{u}=\frac{\vec{m}}{\rho}$ is the velocity of the flows.

This system describes the dynamic behavior of many important physical flows including the propagation of electrons in submicron semiconductor devices (cf. $[4,20,24,37]$ ) and the biological transport of ions for channel proteins (cf. [5] and the references cited therein). In the hydrodynamic model for semiconductor devices, $\rho, \vec{m}$, and $\phi$ are the electron density, the current mass, and the electrostatic potential, 
respectively. In this model, the velocity overshoot and ballistic effects are simulated, which can not be recovered from the classical drift-diffusion model (cf. $[2,3]$ ). The biological model describes the transport of ions between the extracellular side and the cytoplasmic side of the membrane in the ion channels, which are porous proteins inserted across cell membranes. In this case, $\rho, \vec{m}$, and $\phi$ are the ion concentration, the ion translational mass, and the electric potential, respectively.

For concreteness, in this paper we focus on the following initial-boundary problem for (1.1) in the domain $\left\{(\vec{x}, t) \in \mathbf{R}^{N} \times \mathbf{R}_{+}|1 \leqq| \vec{x} \mid \leqq 2\right\}$ :

$$
\left\{\begin{array}{l}
\left.(\rho, \vec{m})\right|_{t=0}=\left(\rho_{0}(\vec{x}), \vec{m}_{0}(\vec{x})\right), \\
\left.\vec{m} \cdot \vec{x}\right|_{|\vec{x}|=1}=\left.\vec{m} \cdot \vec{x}\right|_{|\vec{x}|=2}=0, \\
\left.\phi\right|_{|\vec{x}|=1}=\phi_{1}(t),\left.\quad \phi\right|_{|\vec{x}|=2}=\phi_{2}(t),
\end{array}\right.
$$

where $\phi_{1}$ and $\phi_{2}$ are bounded in $L^{\infty}$. The ideas and methods developed here can be applied to solving the unbounded domain $\left\{(\vec{x}, t) \in \mathbf{R}^{N} \times \mathbf{R}_{+}|| \vec{x} \mid \geqq 1\right\}$ to obtain the similar results by following the techniques as in Chen-Glimm $[9,10]$.

The system (1.1) supports the formation of nonlinear shock and contact waves. Therefore one can not expect the global smooth solutions with large amplitude and has to investigate the weak solutions containing the shock waves. In this paper we develop a shock capturing scheme and apply this scheme to proving new existence results of weak entropy solutions with geometrical structure of the Euler-Poisson equations.

The system (1.1) with spherical symmetry can be rewritten as:

$$
\left\{\begin{array}{l}
\rho_{t}+m_{x}=a(x) m, \\
m_{t}+\left(\frac{m^{2}}{\rho}+p(\rho)\right)_{x}=a(x) \frac{m^{2}}{\rho}+\rho \phi_{x}-\frac{m}{\tau}, \\
\phi_{x x}=a(x) \phi_{x}+\rho-D(x),
\end{array}\right.
$$

where $a(x)=-\frac{N-1}{x}$ and the force term $\phi_{x}$ is nonlocal involving the global behavior of the solution.

In the case $N=1, a(x)=0$, that is, (1.3) becomes the one-dimensional EulerPoisson system as (1.1) without the geometrical source terms. There have been some important investigations on the one-dimensional case, for example, $[1,14,21]$ for the stationary problem and $[36,44,19,29]$ for the evolution problem. In DegondMarkowich [14], the existence for the one-dimensional steady-state equations was obtained in the subsonic case, and in Gamba [21], a viscosity method was used to study the boundary layers that appear when the viscosity coefficient vanishes. In Marcati-Natalini [36] and Zhang [44], the Godunov scheme and the fractional step procedure (cf. [16]) were used to construct the approximate solutions, where the Riemann solutions serve as the building blocks, and a compensated compactness framework (cf. $[6,15]$; also see $[7,17]$ ) was applied to proving the convergence of approximate solutions and the existence of weak solutions. In Fang-Ito [19], the existence of the global weak solution was established by the viscosity method with the aid of the same compensated compactness framework. Also see $[11,12,22,29,38,45]$ for other related references.

In the case $N>1, a(x)$ is not equal to zero. Then the nonlinear resonance between characteristic modes and the geometrical source terms occurs at the sonic state, that is, some of the characteristic speeds and the source speed coincide at the sonic state. Such a nonlinear resonance causes extra difficulties in solving the 
equations (cf. $[9,33,34]$ and the references therein). Therefore more efficient methods have to be developed to solve (1.3) for this case, instead of using the traditional method for the case $a(x)=0$ (see [9, 10, 18, 26, 27, 33, 39, 40,31]). Liu [33] first combined the steady-state solutions with Glimm's random choice method to prove the existence of global solutions of transonic nozzle flow provided the initial data have a small total variation and are bounded away from sonic and vacuum states. In Glaz-Liu [26] a shock capturing scheme was developed to calculate the transient gas flows in a Laval nozzle. Also see Glimm-Marshall-Plohr [27] and EmbidGoodman-Majda [18] for related discussions. Recently, an efficient shock capturing scheme was developed in Chen-Glimm $[9,10]$ to solve the Euler equations with geometrical structure. In $[9,10]$ the approximate solutions were constructed by incorporating the steady-state solutions with the Godunov scheme and the initial data can be arbitrarily large with $L^{\infty}$ bounds. The existence of global weak entropy solutions for general $L^{\infty}$ initial data was established by first discovering the important properties of the steady-state solutions. In this paper, we focus on the system (1.3) with bounded $a(x)$ and nonlocal source terms, which excludes the singularity near $x=0$ for the spherical symmetric case as in the earlier work, but we are able to handle the difficulties from the nonlinear resonance of the system and the nonlocal behavior of the source terms. We use the shock capturing ideas from Chen-Glimm $[9,10]$ to develop an efficient shock capturing scheme for the present problem (1.2) -(1.3). Then we use this scheme to establish the new existence theorem for the global weak solution to the initial-boundary problems of (1.1)-(1.2) with nonlocal source terms, with the aid of the compensated compactness framework.

The general strategy to solve nonlinear problems, such as (1.3), is the following: First one constructs an appropriate sequence of approximate solutions and then makes some apriori estimates, which are good enough to ensure the convergence of a subsequence to the solution. In this process some theorems or frameworks on convergence should be properly chosen based on the estimates one can obtain from the approximate solutions. For (1.3) with $a(x) \neq 0$, as usual, one may consider the Helly framework: the uniform boundedness of the approximate solutions in both $L^{\infty}$ and total variation norms implies the convergence. However, the total variation norm increases as $\rho$ approaches 0 , due to the nonlinear resonance at the vacuum state $\rho=0$, that is, different characteristic speeds coincide at the vacuum, as well as the nonlocal forcing term and the geometrical source terms.

This difficulty in applying the Helly compactness framework to (1.3) can be overcome by using the compensated compactness framework: the uniform boundedness of the approximate solutions and $H^{-1}$ compactness of the corresponding weak entropy dissipation measures implies the compactness of the approximate solutions. The importance of this framework is that it takes the vacuum into account in the correct physical variables $(\rho, m)$ near the vacuum. This framework was proved in [17] for the case $\gamma=1+\frac{2}{2 m+1}, m \geqq 2$ integers, and in [6] for the general case of gases $1<\gamma \leqq 5 / 3$ (also see [15,7]). Further discussions on this framework for other cases can be found in [32]. Due to the geometrical source terms, we adopt the approach of Chen-Glimm $[9,10]$ to construct the approximate solutions satisfying the framework. One of the key ideas of this approach is to use the piecewise steady-state solutions, which incorporate the geometrical source terms, to replace the piecewise constants from the Riemann solutions as the building blocks, motivated by $[18,26,27,31,33,39,40]$. The main difficulty to achieve this idea is that, in the transonic case, no smooth steady-state solution exists and an approximate steady-state solution, including a standing shock, has to be introduced, satisfying 
some important properties similar to those of the smooth solution. This construction considerably improves the traditional fractional Godunov scheme for this case. The corresponding approximate solutions can be estimated to satisfy the required bounds. For this purpose, the properties of the steady-state solutions are important, such as the oscillation and the average of the steady-state solutions and the behavior of the corresponding Riemann invariants, since the steady-state solutions are the fundamental building blocks. See Chen-Glimm $[9,10]$ for the details.

To construct the approximate solutions of (1.3), we also incorporate the fractional step procedure for the extra terms like $\rho \phi_{x}-\frac{m}{\tau}$ into our construction of approximate solutions, and some careful attention must be paid to the nonlocal term $\rho \phi_{x}$ as in Marcati-Natalini [36] and Zhang [44]. In the level of the fractional step procedure, our fundamental building blocks are the steady-state solutions, while the building blocks in Marcati-Natalini [36] and Zhang [44] are the classical Riemann solutions. First we solve the third equation in (1.3) to get $\phi_{x}$ that involves the term $\int_{1}^{x} \rho d \xi$. To obtain the uniform bound of the approximate solutions, we estimate the Riemann invariants, for which the nonlocal term $\int_{1}^{2} \rho^{h} d x$ is involved. For the case $a(x)=0$, one has the conservation of particles, i.e., $\int \rho^{h}(x, t) d x=\int \rho_{0}(x) d x$, as used and proved in Marcati-Natalini [36] and Zhang [44]. For the case $a(x) \neq 0$, one does not have such a conservation principle because of the geometrical source terms. Therefore we have to make a proper estimate on the nonlocal term in order to get the uniform estimate of the approximate solutions. For this purpose, we use the first equation of (1.3) in a different but equivalent way. We change the definition of the Godunov values in the scheme and prove a new property of the steady-state solution. Then we can make a new estimate on the nonlocal term, which is good enough so that the uniform estimate can be achieved. To estimate the $H^{-1}$ compactness of the weak entropy dissipation measures, we first make the estimates on the mechanical entropy pair, which will also be used later to prove the convergence and existence. Because of the different definition of the Godunov values, we have to prove the existence separately for the first and the second equations of (1.3) and to use the new property of the steady-state solution. Some extra terms from the fractional step procedure have to be taken into account.

We also apply these procedures to prove the existence of the two-dimensional symmetric rotating solutions of (1.1) with the aid of the method of quasidecoupling (cf. [8]), due to the linear degeneracy of the extra field generated from the angular momentum and the fractional step procedure. We first transform the system into another equivalent system, for which we can conveniently make the uniform estimates of the approximate solutions. Then we show the convergence of the approximate solutions $\left(\rho^{h}, m^{h}\right)$ by the compensated compactness framework. To show the convergence of $\tilde{m}^{h}$, we apply the method of quasidecoupling to deduce that the family of the Young measures, determined by the approximate solutions, is the family of Dirac measures.

We will also investigate the boundary values of the weak solutions as in [28]. First we define the traces of the weak solutions along the boundary, which satisfy the weak form of the Gauss-Green theorem and have the Lipschitz regularity. Then we prove that the weak solutions satisfy the initial-boundary conditions, which also tells us the well-posedness of the boundary problem.

In Sect. 2, we review some basic properties of the Riemann solutions to the homogeneous systems. In Sect. 3, we first revisit some important facts on the steadystate solutions (see $[9,10]$ for more details). Then we prove a new property of the 
steady-state solutions, which is essential to estimate the nonlocal term and to prove the existence.

In Sect. 4, we combine the modified first order Godunov scheme with the fractional step procedure to construct the approximate solutions for the initial-boundary problems. The steady-state solutions are the building blocks in our construction. Some estimates on the entropy are also given.

In Sect. 5, we first discuss the spherically symmetrical solutions and prove the uniform boundedness of the approximate solutions in Sect. 5.1 and $H^{-1}$ compactness of the corresponding entropy dissipation measures in Sect. 5.2. The convergence of the approximate solutions and the existence of weak entropy solution are established in Sect. 5.3. Then we apply this theory to obtain the existence of the nozzle solutions.

In Sect. 6, we apply this existence theory to the two-dimensional symmetric rotating solutions with the aid of the method of quasidecoupling (cf. [8]) and a transform of the system into an equivalent one. In Sect. 7, we investigate the traces of the weak solutions on the boundary.

We remark that all the uniform bounds of the approximate solutions we obtain are independent of the momentum relaxation time $\tau$, which is important for investigating other problems such as the relaxation limit as $\tau \rightarrow 0$. We also note that the Godunov scheme in this paper can be replaced by the Lax-Friedrich scheme. The techniques and ideas developed here can be applied to solving the bipolar EulerPoisson equations (cf. $[4,41]$ ) with geometrical structure in several space variables. For recent developments on two-dimensional viscous steady-state solutions for the Euler-Poisson equations, see Gamba-Morawetz [23].

\section{Preliminaries}

In this section, we review some basic facts about the Riemann solutions for the homogeneous systems. The equations for the two-dimensional symmetric rotating flow discussed later are also considered here, parallel to the equations for the spherically symmetric flow.

Consider the homogeneous system:

$$
v_{t}+f(v)_{x}=0, \quad 1<x<2,
$$

where $v=(\rho, \bar{m})^{\top}$ and $f(v)$ take one of the following two forms:

or

$$
\bar{m}=m, \quad f(v)=\left(m, \frac{m^{2}}{\rho}+p(\rho)\right)^{\top},
$$

$$
\bar{m}=(m, \hat{m})^{\top}, \quad f(v)=\left(m, \frac{m^{2}}{\rho}+p(\rho), \frac{m \hat{m}}{\rho}\right)^{\top}
$$

with $p(\rho)=\rho^{\gamma} / \gamma, \gamma>1$.

The systems for (2.2) and (2.3) have parallel facts on the Riemann solutions. The eigenvalues are

for (2.2) and

$$
\lambda_{1}=\frac{m}{\rho}-\rho^{\theta}, \quad \lambda_{2}=\frac{m}{\rho}+\rho^{\theta},
$$

$$
\lambda_{1}=\frac{m}{\rho}-\rho^{\theta}, \quad \lambda_{2}=\frac{m}{\rho}+\rho^{\theta}, \quad \lambda_{3}=\frac{m}{\rho},
$$


for (2.3), where $\theta=\frac{\gamma-1}{2}$. The two characteristic fields for (2.2), and the first and the second characteristic fields for (2.3) are genuinely nonlinear. The third characteristic field for (2.3) is linearly degenerate.

The Riemann invariants are

for (2.2) and

$$
w=\frac{m}{\rho}+\frac{\rho^{\theta}}{\theta}, \quad z=\frac{m}{\rho}-\frac{\rho^{\theta}}{\theta},
$$

$$
w=\frac{m}{\rho}+\frac{\rho^{\theta}}{\theta}, \quad z=\frac{m}{\rho}-\frac{\rho^{\theta}}{\theta}, \quad \omega=\frac{\hat{m}}{\rho},
$$

for (2.3).

The discontinuity in the weak solutions of (2.1) satisfies the Rankine-Hugoniot condition:

$$
\sigma\left(v-v_{0}\right)=f(v)-f\left(v_{0}\right),
$$

where $\sigma$ is the propagation speed of the discontinuity, and $v_{0}$ and $v$ are the corresponding left state and right state, respectively. A discontinuity is a shock if it satisfies the entropy condition:

$$
\sigma\left(\eta(v)-\eta\left(v_{0}\right)\right)-\left(q(v)-q\left(v_{0}\right)\right) \geqq 0,
$$

for any convex entropy pair $(\eta, q)$. The shock with speed $\sigma=0$ is called the standing shock.

Consider the Riemann problem of (2.1) with initial data

$$
\left.v\right|_{t=0}= \begin{cases}v_{-}, & x<x_{0}, \\ v_{+}, & x>x_{0},\end{cases}
$$

where $x_{0} \in(1,2), v_{ \pm}=\left(\rho_{ \pm}, \bar{m}_{ \pm}\right)^{\top}$, and $\rho_{ \pm} \geqq 0$ and $\bar{m}_{ \pm}$are constants satisfying $\left|\frac{\bar{m}_{ \pm}}{\rho_{ \pm}}\right|<\infty$. For the Riemann problem of $(2.2)$, there are two distinct types of rarefaction waves and shock waves. For the Riemann problem of (2.3), there is a linear wave - the contact discontinuity for $\lambda_{3}$, in addition to the rarefaction waves and shock waves corresponding to $\lambda_{i}, i=1,2$, on which $\hat{m}=$ const.

For the Riemann problem (2.6) and the Riemann initial-boundary problem of (2.1) with data:

$$
\left.v\right|_{t=0}=v_{+},\left.\quad m\right|_{x=1}=0,
$$

we have the following facts on the solutions.

Lemma 2.1. There exists a piecewise smooth entropy solution $v(x, t)$ for each problem of (2.6) and (2.7) satisfying

$$
\left\{\begin{array}{l}
w(v(x, t)) \leqq \max \left(w\left(v_{-}\right), w\left(v_{+}\right)\right), \\
w(v(x, t))-z(v(x, t)) \geqq 0,
\end{array}\right.
$$

and, for (2.6),

$$
z(v(x, t)) \geqq \min \left(z\left(v_{-}\right), z\left(v_{+}\right)\right),
$$

and, for (2.7),

$$
z(v(x, t)) \geqq \min \left(z\left(v_{+}\right), 0\right)
$$


In addition, for these two problems for (2.3),

$$
\min \left(\omega_{-}, \omega_{+}\right) \leqq \omega(x, t) \leqq \max \left(\omega_{-}, \omega_{+}\right) .
$$

Lemma 2.2. For the Riemann problem (2.6), the regions

and

$$
\sum=\left\{(\rho, m): w \leqq w_{0}, z \geqq z_{0}, w-z \geqq 0\right\}
$$

$$
\sum=\left\{(\rho, m, \hat{m}): w \leqq w_{0}, z \geqq z_{0}, w-z \geqq 0, \omega_{-} \leqq \omega \leqq \omega_{+}\right\}
$$

are invariant for (2.2) and (2.3), respectively. For the Riemann initial-boundary problem (2.7), the regions

$$
\sum=\left\{(\rho, m): w \leqq w_{0}, z \geqq z_{0}, w-z \geqq 0\right\}, \quad z_{0} \leqq 0 \leqq \frac{w_{0}+z_{0}}{2},
$$

and

$\sum=\left\{(\rho, m, \hat{m}): w \leqq w_{0}, z \geqq z_{0}, w-z \geqq 0, \omega_{-} \leqq \omega \leqq \omega_{+}\right\}, \quad z_{0} \leqq 0 \leqq \frac{w_{0}+z_{0}}{2}$,

are invariant for (2.2) and (2.3), respectively. That is, if the Riemann data lie in $\sum$, then the Riemann solutions $v(x, t) \in \sum$ and $\frac{1}{b-a} \int_{a}^{b} v(x, t) d x \in \sum$.

For the Riemann initial-boundary problem of (2.1) with data: $\left.v\right|_{t=0}=v_{-}$, $\left.m\right|_{x=2}=0$, we have the similar results to those for (2.7) in the above two lemmas.

A pair of mappings $(\eta, q): \mathbf{R}^{2} \rightarrow \mathbf{R}^{2}$ is called an entropy-entropy flux pair if $\nabla q=\nabla \eta \nabla f$. If $\tilde{\eta}(\rho, \bar{u}) \equiv \eta(\rho, \rho \bar{u})$ satisfies $\tilde{\eta}(0, \bar{u})=0$, for any fixed $\bar{u}=\frac{\bar{m}}{\rho}$, then $\eta$ is called a weak entropy. For example, the mechanical energy-energy flux pair

$$
\eta_{*}=\frac{1}{2} \frac{\bar{m}^{2}}{\rho}+\frac{1}{\gamma(\gamma-1)} \rho^{\gamma}, \quad q_{*}=m\left(\frac{1}{2} \frac{\bar{m}^{2}}{\rho^{2}}+\frac{\rho^{\gamma-1}}{\gamma-1}\right),
$$

is a strictly convex weak entropy pair of (2.1).

Lemma 2.3. Assume $(\rho, \bar{m})^{\top}=(\rho, \rho \bar{u})^{\top}$ is a Riemann solution of (2.1), and $0 \leqq \rho \leqq C^{\prime},|\bar{u}| \leqq C^{\prime}$, for some constant $C^{\prime}>0$. Then, for any weak entropy pair $(\eta, q)$,

$$
\begin{aligned}
& |\nabla \eta| \leqq C,|\nabla q| \leqq C ; \quad\left|v^{\top} \nabla^{2} \eta v\right| \leqq C v^{\top} \nabla^{2} \eta_{*} v ; \\
& |\sigma[\eta]-[q]| \leqq C\left(\sigma\left[\eta_{*}\right]-\left[q_{*}\right]\right),
\end{aligned}
$$

where $v$ is any vector and the constant $C$ depends only on $C^{\prime}$ and $\eta$, independent of $v$.

\section{Steady-State Solutions}

In this section, we first revisit some important properties of the steady-state solutions from $[9,10]$. Then we prove a new lemma that is essential to our uniform estimates and existence in Sect. 5 due to the geometrical source terms and the nonlocal term. As in Sect. 2, the equations for the two-dimensional symmetric rotating flow discussed later are also considered here, parallel to the equations for the spherically symmetric flow. 
Consider the system of steady-state equations with boundary condition:

$$
\left\{\begin{array}{l}
f(v)_{x}=a(x) g(v) \\
\left.v\right|_{x=x_{0}}=v_{0}
\end{array}\right.
$$

where $a(x)=-\frac{A^{\prime}(x)}{A(x)}$ with $A(x) \in C^{2}, A(x) \geqq c_{0}>0$, and $v=(\rho, \bar{m})^{\top}, \quad v_{0}=$ $\left(\rho_{0}, \bar{m}_{0}\right)^{\top}, f(v)$ and $g(v)$ take one of the following two forms:

$$
\left\{\begin{array}{l}
\bar{m}=m=\rho u, \quad \bar{m}_{0}=m_{0}=\rho_{0} u_{0}, \\
f(v)=\left(m, \frac{m^{2}}{\rho}+p(\rho)\right)^{\top}, \quad g(v)=\left(m, \frac{m^{2}}{\rho}\right)^{\top},
\end{array}\right.
$$

or

$$
\left\{\begin{array}{l}
\bar{m}=(m, \hat{m})^{\top}=(\rho u, \rho \omega)^{\top}, \quad \bar{m}_{0}=\left(m_{0}, \hat{m}_{0}\right)^{\top}=\left(\rho_{0} u_{0}, \rho_{0} \omega_{0}\right)^{\top}, \\
f(v)=\left(m, \frac{m^{2}}{\rho}+p(\rho), \frac{m \hat{m}}{\rho}\right)^{\top}, \quad g(v)=\left(m, \frac{m^{2}}{\rho}, \frac{2 m \hat{m}}{\rho}\right)^{\top} .
\end{array}\right.
$$

The steady-state solutions for (3.2) and (3.3) have parallel properties. Set the sound speed $c=\rho^{\theta}$. Then $M=M(v(x))=\frac{u(x)}{c(x)}$ is the Mach number and $M_{0}=M\left(v_{0}\right)$.

For the nonsonic case, $\left|M_{0}^{2}-1\right| \geqq h^{\beta} M_{0}^{2}$, with some $\beta \in\left(0, \frac{1}{6}\right)$ and $h \in\left(0, h_{0}\right)$ for some sufficiently small $h_{0} \in(0,1)$, (3.1) has a smooth solution.

When $\left|M_{0}^{2}-1\right| \leqq h^{\beta} M_{0}^{2}$, the steady-state equation (3.1) does not have exact smooth solutions, but has approximate solutions in the following sense.

Definition 3.1. $v$ is an approximate solution of (3.1) if

$$
\left|f(v)_{x}-a(x) g(v)\right| \leqq o(1), \quad \text { as } h \rightarrow 0 .
$$

Near the sonic case, $K_{0} \sqrt{h} \leqq\left|M_{0}^{2}-1\right| \leqq h^{\beta} M_{0}^{2}$, with $K_{0}=2 \sqrt{\frac{\|a\|_{C}}{\theta}}$, take

$$
\left\{\begin{array}{l}
\rho(x)=\rho_{0}\left(1+\frac{M_{0}^{2}-1}{2(\theta+1)}\left(1-\sqrt{1-\frac{4(\theta+1) a_{0}}{\left(M_{0}^{2}-1\right)^{2}}(x-\tilde{x})}\right)\right), \\
m(x)=m_{0}\left(1+a_{0}\left(x-x_{0}\right)\right), \quad \hat{m}(x)=\frac{a(x)}{a_{0}} \omega_{0} \rho(x),
\end{array}\right.
$$

where $a_{0}=a\left(x_{0}\right), \tilde{x} \in\left(x_{0}-\frac{h}{2}, x_{0}+\frac{h}{2}\right)$. Then $v=(\rho, m)^{\top}$ for $(3.2)$, or $v=(\rho, m, \hat{m})^{\top}$ for (3.3), is an approximate solution in the sense of (3.4) and satisfies

$$
f(v)_{x}-a(x) g(v)=O\left(h^{\beta}\right) .
$$

For the transonic case, $\left|M_{0}^{2}-1\right|<K_{0} \sqrt{h}$, we introduce a standing shock at $x=\tilde{x}$ with left state $v_{-}=\left(\rho_{0-}, \bar{m}_{0}\right)$ and right state $v_{+}=\left(\rho_{0+}, \bar{m}_{0}\right)$, where

$$
\rho_{0 \pm}=\rho_{0}\left(\frac{\theta M_{0}+1}{\theta+1}\right)^{\frac{1}{\theta}}\left(1 \pm K_{0} \sqrt{h}\right) .
$$

The corresponding Mach numbers are $M_{0 \pm}^{2}=1 \mp 2(\theta+1) K_{0} \sqrt{h}+O(h)$. Take

$$
\rho_{ \pm}(x)=\rho_{0 \pm}\left(1+\frac{M_{0 \pm}^{2}-1}{2(\theta+1)}\left(1-\sqrt{1-\frac{4(\theta+1) a_{0}}{\left(M_{0 \pm}^{2}-1\right)^{2}}(x-\tilde{x})}\right)\right),
$$


with $\quad \tilde{x} \in\left(x_{0}-\frac{2+\theta}{4(1+\theta)} h, x_{0}+\frac{2+\theta}{4(1+\theta)} h\right)$. Then $v(x)=(\rho(x), m(x))^{\top}$ for (3.2), or $v(x)=(\rho(x), m(x), \tilde{m}(x))^{\top}$ for $(3.3)$, defined by

$$
\left\{\begin{array}{l}
\rho(x)=\left\{\begin{array}{l}
\rho_{-}(x), \quad x \in\left[x_{0}-\frac{h}{2}, \tilde{x}\right), \\
\text { standing shock, } \quad x=\tilde{x}, \\
\rho_{+}(x), \quad x \in\left(\tilde{x}, x_{0}+\frac{h}{2}\right],
\end{array}\right. \\
m(x)=m_{0}\left(1+a_{0}\left(x-x_{0}\right)\right), \quad \hat{m}(x)=\frac{a(x)}{a_{0}} \omega_{0} \rho(x),
\end{array}\right.
$$

is an approximate solution of (3.1) with $\rho_{0} \geqq 0$ in the sense of (3.4) satisfying (3.6). Furthermore, we have

Lemma 3.1. There exists a smooth steady-state solution $v(x)$ of (3.1) when $\left|M_{0}^{2}-1\right| \geqq h^{\beta} M_{0}^{2}$, an approximate smooth steady-state solution $v(x)$ when $K_{0} \sqrt{h} \leqq\left|M_{0}^{2}-1\right| \leqq h^{\beta} M_{0}^{2}$, and an approximate steady-state solution including a standing shock at some $\tilde{x} \in\left(x_{0}-\frac{2+\theta}{4(1+\theta)} h, x_{0}+\frac{2+\theta}{4(1+\theta)} h\right)$ when $\left|M_{0}^{2}-1\right|<K_{0} \sqrt{h}$, with $h \leqq h_{0}$, in the sense of (3.4), such that, for $x \in\left[x_{0}-\frac{h}{2}, x_{0}+\frac{h}{2}\right]$,

$$
\begin{gathered}
\left\{\begin{array}{l}
\rho(x) \geqq 0, \\
v(x)=v_{0}(1+O(\sqrt{h})),
\end{array}\right. \\
\left\{\begin{array}{l}
w(v(x)) \leqq w\left(v_{0}\right)(1+C h), \quad \text { if } M_{0}>0, \\
z(v(x)) \geqq z\left(v_{0}\right)(1+C h), \quad \text { if } M_{0}<0,
\end{array}\right.
\end{gathered}
$$

and, for (3.2),

$$
\frac{1}{h} \int_{x_{0}-\frac{h}{2}}^{x_{0}+\frac{h}{2}} v(x) d x=v_{0}\left(1+O\left(h^{2(1-\beta)}\right)\right),
$$

and, for (3.3),

$$
\left\{\begin{array}{l}
\frac{1}{h} \int_{x_{0}-\frac{h}{2}}^{x_{0}+\frac{h}{2}} v(x) d x=v_{0}\left(1+O\left(h^{1+\beta}\right)\right), \\
\left|\omega(x)-\omega_{0}\right| \leqq\left|\omega_{0}\right|\left|x-x_{0}\right|
\end{array}\right.
$$

where the constant $C$ and the bounds $O(\sqrt{h}), O\left(h^{2(1-\beta)}\right)$, and $O\left(h^{1+\beta}\right)$ depend only on the bound of $A(x)$ and are independent of $M_{0}$.

We now introduce a new property of the steady-state solution.

Lemma 3.2. Let $v(x)=(\rho(x), \bar{m}(x))$ be a smooth steady-state solution of (3.1) when $\left|M_{0}^{2}-1\right| \geqq h^{\beta} M_{0}^{2}$, or an approximate smooth steady-state solution of (3.1) when $\left|M_{0}^{2}-1\right| \leqq h^{\beta} M_{0}^{2}$ in Lemma 3.1. Then, for $h \leqq h_{0}, \rho(x)$ satisfies the following property:

(1) If $\left|M_{0}^{2}-1\right| \geqq h^{\beta} M_{0}^{2}$,

$$
\frac{1}{h} \int_{x_{0}-\frac{h}{2}}^{x_{0}+\frac{h}{2}} A(x)\left(\rho(x)-\rho_{0}\right) d x=\rho_{0} O\left(h^{2(1-\beta)}\right) ;
$$


(2) If $\left|M_{0}^{2}-1\right| \leqq h^{\beta} M_{0}^{2}$,

$$
\frac{1}{h} \int_{x_{0}-\frac{h}{2}}^{x_{0}+\frac{h}{2}} A(x)\left(\rho(x)-\rho_{0}\right) d x=\rho_{0} O\left(h^{1+\beta}\right),
$$

where the bounds $O\left(h^{2(1-\beta)}\right)$ and $O\left(h^{1+\beta}\right)$ depend only on the bound of $A(x)$.

Proof. (1) If $\left|M_{0}^{2}-1\right| \geqq h^{\beta} M_{0}^{2}$, then (3.1) has a smooth solution $v(x)=$ $(\rho(x), \bar{m}(x))$. By the Taylor expansion on $\rho(x)$, for $x \in\left[x_{0}-\frac{h}{2}, x_{0}+\frac{h}{2}\right]$,

$$
A(x)\left(\rho(x)-\rho_{0}\right)=\left(A\left(x_{0}\right)+\left(A(x)-A\left(x_{0}\right)\right)\right) \rho^{\prime}\left(x_{0}\right)\left(x-x_{0}\right)+\frac{1}{2} A(x) \rho^{\prime \prime}(y)\left(x-x_{0}\right)^{2},
$$

for some $y$ between $x_{0}$ and $x$. A careful estimate shows that (cf. [9])

$$
\left|\rho^{\prime}(x)\right| \leqq C \rho_{0} h^{-\beta}, \quad\left|\rho^{\prime \prime}(x)\right| \leqq C \rho_{0} h^{-2 \beta}
$$

Therefore

$$
\begin{aligned}
\frac{1}{h} \int_{x_{0}-\frac{h}{2}}^{x_{0}+\frac{h}{2}} A(x)\left(\rho(x)-\rho_{0}\right) d x & =A\left(x_{0}\right) \rho^{\prime}\left(x_{0}\right) \frac{1}{h} \int_{x_{0}-\frac{h}{2}}^{x_{0}+\frac{h}{2}}\left(x-x_{0}\right) d x+\rho_{0} O\left(h^{2-2 \beta}\right) \\
& =\rho_{0} O\left(h^{2(1-\beta)}\right) .
\end{aligned}
$$

(2) If $\left|M_{0}^{2}-1\right| \leqq h^{\beta} M_{0}^{2}$, (3.1) has an approximate solution (3.5) or (3.8) satisfying $\rho(x)-\rho_{0}=\rho_{0} O(\sqrt{h})$. Then by (3.9), (3.10), and $\beta \in\left(0, \frac{1}{6}\right)$, we conclude

$$
\begin{aligned}
\frac{1}{h} \int_{x_{0}-\frac{h}{2}}^{x_{0}+\frac{h}{2}} A(x)\left(\rho(x)-\rho_{0}\right) d x & =\frac{1}{h} \int_{x_{0}-\frac{h}{2}}^{x_{0}+\frac{h}{2}}\left(A\left(x_{0}\right)\left(\rho(x)-\rho_{0}\right)\right. \\
& \left.+\left(A(x)-A\left(x_{0}\right)\right)\left(\rho(x)-\rho_{0}\right)\right) d x \\
& =\rho_{0} O\left(h^{1+\beta}\right) .
\end{aligned}
$$

This completes the proof.

\section{The Shock Capturing Scheme}

Consider the following problem:

$$
\left\{\begin{array}{l}
v_{t}+f(v)_{x}=a(x) g(v)+G(v, x, t), \quad 1<x<2, \\
\left.v\right|_{t=0}=v_{0}(x) \\
\left.m\right|_{x=1}=\left.m\right|_{x=2}=0
\end{array}\right.
$$

where $v=(\rho, \bar{m})^{\top}$ with $\bar{m}=m$, or $\bar{m}=(m, \hat{m})^{\top}, f(v), g(v)$ and $a(x)$ are the same as in $(3.1)$, and $G=\left(0, \bar{G}_{2}\right) \in C$.

In this section, we construct the approximate solutions $v^{h}=\left(\rho^{h}, \bar{m}^{h}\right)^{\top}=$ $\left(\rho^{h}, \rho^{h} \bar{u}^{h}\right)^{\top}$ of (4.1) in the strip $0 \leqq t \leqq T$ for any fixed $T \in(0, \infty)$, where $h=\frac{1}{M}>0$ with a large positive integer $M$ and $\Delta t>0$ are the space mesh 
length and the time mesh length, respectively, and satisfy the following CourantFriedrichs-Levy condition:

$$
\Lambda=\max \left(\sup _{0 \leqq t \leqq T}\left|\lambda_{i}\left(\rho^{h}, m^{h}\right)\right|\right) \leqq \frac{\gamma-1}{4(\gamma+1)} \frac{h}{\Delta t} \leqq 2 \Lambda,
$$

where $\lambda_{i}, i=1,2$, or 3 , are the eigenvalues of (4.1).

Assume that $v^{h}(x, t)$ is defined for $t<n \Delta t$. Then we define $v_{j}^{n}=\left(\rho_{j}^{n}, \bar{m}_{j}^{n}\right)$ as:

$$
\rho_{j}^{n}=\frac{\int_{1+\left(j-\frac{1}{2}\right) h}^{1+\left(j+\frac{1}{2}\right) h} A(x) \rho^{h}(x, n \Delta t-0) d x}{\int_{1+\left(j-\frac{1}{2}\right) h}^{1+\left(j+\frac{1}{2}\right) h} A(x) d x}, \quad \bar{m}_{j}^{n}=\frac{1}{h} \int_{1+\left(j-\frac{1}{2}\right) h}^{1+\left(j+\frac{1}{2}\right) h} \bar{m}^{h}(x, n \Delta t-0) d x,
$$

for $2 \leqq j \leqq M-2$; and

$$
\begin{aligned}
\rho_{1}^{n} & =\frac{\int_{1}^{1+\frac{3}{2} h} A(x) \rho^{h}(x, n \Delta t-0) d x}{\int_{1}^{1+\frac{3}{2} h} A(x) d x}, \quad \bar{m}_{1}^{n}=\frac{2}{3 h} \int_{1}^{1+\frac{3}{2} h} \bar{m}^{h}(x, n \Delta t-0) d x ; \\
\rho_{M-1}^{n} & =\frac{\int_{2-\frac{3}{2} h}^{2} A(x) \rho^{h}(x, n \Delta t-0) d x}{\int_{2-\frac{3}{2} h}^{2} A(x) d x}, \quad \bar{m}_{M-1}^{n}=\frac{2}{3 h} \int_{2-\frac{3}{2} h}^{2} \bar{m}^{h}(x, n \Delta t-0) d x .
\end{aligned}
$$

In the strip $n \Delta t \leqq t<(n+1) \Delta t$, we define $v_{0}^{h}(x, t)$ as follows:

(a) For $1+j h \leqq x \leqq 1+(j+1) h, 1 \leqq j \leqq M-2, v_{0}^{h}(x, t)$ is the approximate solution of the generalized Riemann problem of the system

with initial data

$$
v_{t}+f(v)_{x}=a(x) g(v),
$$

$$
\left.v\right|_{t=n \Delta t}= \begin{cases}v_{-}(x), & x<1+\left(j+\frac{1}{2}\right) h, \\ v_{+}(x), & x>1+\left(j+\frac{1}{2}\right) h,\end{cases}
$$

where $v_{-}(x)$ and $v_{+}(x)$ are the smooth solutions or approximate solutions in Sect. 3 of the steady-state equation

$$
f(v)_{x}=a(x) g(v),
$$

with boundary conditions: $v_{-}(1+j h)=v_{j}^{n}, v_{+}(1+(j+1) h)=v_{j+1}^{n}$;

(b) For $1 \leqq x \leqq 1+h, v_{0}^{h}(x, t)$ is the approximate solution of the generalized Riemann initial-boundary problem of (4.2) with data

$$
\left.v\right|_{t=n \Delta t}=v_{1}^{+}(x),\left.\quad m\right|_{x=1}=0,
$$

where $v_{1}^{+}(x)$ is the smooth solution or the approximate solution in Sect. 3 of the steady-state equation (4.3) with boundary condition: $v_{1}^{+}(1+h)=v_{1}^{n}$;

(c) For $2-h \leqq x \leqq 2, v_{0}^{h}(x, t)$ is the approximate solution of the generalized Riemann initial-boundary problem of (4.2) with data:

$$
\left.v\right|_{t=n \Delta t}=v_{M-1}^{-}(x),\left.\quad m\right|_{x=2}=0,
$$

where $v_{M-1}^{-}(x)$ is the smooth solution or the approximate solution in Sect. 3 of the steady-state equation (4.3) with boundary condition: $v_{M-1}^{-}(2-h)=v_{M-1}^{n}$. 
We solve the above problem for small time approximately to get $v_{0}^{h}(x, t)$ by perturbing about the solution $R$ of the corresponding Riemann problem of the homogeneous system:

$$
v_{t}+f(v)_{x}=0
$$

with data

$$
\left.v\right|_{t=n \Delta t}= \begin{cases}v_{-}\left(1+\left(j+\frac{1}{2}\right) h-0\right), & x<1+\left(j+\frac{1}{2}\right) h, \\ v_{+}\left(1+\left(j+\frac{1}{2}\right) h+0\right), & x>1+\left(j+\frac{1}{2}\right) h,\end{cases}
$$

for $1+j h \leqq x<1+(j+1) h, 1 \leqq j \leqq M-2$; and the Riemann initial-boundary problems of (4.6) with data (4.4), for $1<x \leqq 1+h$; and with data (4.5), for $2-h \leqq x<2$.

First, let

$$
R_{a}= \begin{cases}(\rho, \bar{m})=R, & \text { if } \rho(x, t) \geqq h^{\beta}, \\ \left(h^{\beta}, \bar{m}\right), & \text { otherwise }\end{cases}
$$

Then $R_{a}(x, t)$ satisfies the entropy condition on its discontinuities and

$$
\left|R_{a}(x, t)-R(x, t)\right| \begin{cases}=0, & \text { if } \rho(x, t) \geqq h^{\beta}, \\ \leqq C h^{\beta}, & \text { otherwise } .\end{cases}
$$

Then, as in [13], we approximate the possible existing $\kappa^{\text {th }}$ rarefaction waves $\left(v_{-}^{r}, v_{+}^{r}\right), \kappa=1,2$, in $R_{a}(x, t)$ by finite discontinuous rays $\frac{x_{i}}{t}=\lambda_{\kappa}\left(v_{i}^{r}\right)$ separating finite constant states $v_{i}^{r}, i=0,1, \ldots, I_{r}$, with $v_{0}^{r}=v_{-}^{r}$ and $v_{I_{r}}^{r}=v_{+}^{r}$, such that

$$
\begin{aligned}
& \text { if } \kappa=1, w\left(v_{i+1}^{r}\right)=w\left(v_{i}^{r}\right)+h, z\left(v_{i+1}^{r}\right)=z\left(v_{i}^{r}\right), 0 \leqq i \leqq I_{r}-1, \\
& \text { if } \kappa=2, z\left(v_{i+1}^{r}\right)=z\left(v_{i}^{r}\right)+h, w\left(v_{i+1}^{r}\right)=w\left(v_{i}^{r}\right), 0 \leqq i \leqq I_{r}-1 .
\end{aligned}
$$

In this way, we obtain the approximate Riemann solutions consisting of finite discontinuities separating finite constant states $v_{i}, i=0,1, \ldots, I$, with $v_{0}=v_{-}(1+(j+$ $\left.\left.\frac{1}{2}\right) h-0\right)$ and $v_{I}=v_{+}\left(1+\left(j+\frac{1}{2}\right) h+0\right)$. Let $\hat{v}_{i}(x)=\left(\hat{\rho}_{i}(x), \hat{\bar{m}}_{i}(x)\right)$ be the exact smooth or approximate steady-state solutions such that $\hat{v}_{i}\left(1+\left(j+\frac{1}{2}\right) h\right)=v_{i}$.

We use the cut-off technique and denote by $v_{i}(x)=\left(\rho_{i}(x), \rho_{i}(x) \bar{u}_{i}(x)\right)$, $0 \leqq i \leqq I$, the approximate steady-state solutions as follows:

$$
\rho_{i}(x)=\max \left(\hat{\rho}_{i}(x), h^{\beta}\right), \quad \bar{u}_{i}(x)=\frac{\hat{m}_{i}(x)}{\hat{\rho}_{i}(x)}, \quad 0 \leqq i \leqq I .
$$

The approximate solution $v_{0}^{h}(x, t)=\left(\rho_{0}^{h}(x, t), \bar{m}_{0}^{h}(x, t)\right)$ in the rectangle $[1+j h$, $1+(j+1) h] \times[n \Delta t,(n+1) \Delta t)$ or $[1,1+h] \times[n \Delta t,(n+1) \Delta t)$ or $[2-h, 2] \times[n \Delta t$, $(n+1) \Delta t)$ consists of the exact or approximate steady states $v_{i}(x), i=0,1, \ldots, I$, separated by the discontinuities, subject to the Rankine-Hugoniot condition, with speeds

$$
\frac{d x(t)}{d t}=v_{i}(x(t))+(-1)^{\kappa} \sqrt{\frac{\rho_{i+1}(x(t))}{\rho_{i}(x(t))} \frac{p\left(\rho_{i+1}(x(t))\right)-p\left(\rho_{i}(x(t))\right)}{\rho_{i+1}(x(t))-\rho_{i}(x(t))}},
$$

where $\kappa=1$ or $\kappa=2$, determined by the $\kappa^{\text {th }}$ original elementary waves from which the discontinuity comes. Then the approximate solutions $v_{0}^{h}(x, t)$ approach the approximate Riemann solutions as $t \rightarrow n \Delta t$. 
We have the following estimates on the entropy as in $[9,10]$ :

Lemma 4.1. There is a constant $C$ depending only on the uniform bound of $v_{0}^{h}(x, t)$ such that, on any approximate shock wave with speed $\sigma_{i}$,

$$
\sigma_{i}\left(\eta_{*}\left(v_{i+1}\right)-\eta_{*}\left(v_{i}\right)\right)-\left(q_{*}\left(v_{i+1}\right)-q_{*}\left(v_{i}\right)\right) \geqq 0,
$$

and

$$
\begin{aligned}
& \mid \sigma_{i}\left(\eta\left(v_{i+1}(x(t))\right)-\eta\left(v_{i}(x(t))\right)\right)-\left(q\left(v_{i+1}(x(t))\right)-q\left(v_{i}(x(t))\right)\right) \\
& \quad-\left(\sigma_{i}\left(\eta_{*}\left(v_{i+1}\right)-\eta_{*}\left(v_{i}\right)\right)-\left(q_{*}\left(v_{i+1}\right)-q_{*}\left(v_{i}\right)\right)\right) \mid \leqq C h^{\frac{3}{2}-2 \beta}
\end{aligned}
$$

and, on the discontinuous rays, $x=x_{i}(t), \sigma_{i}=\frac{d x_{i}(t)}{d t}$, of the approximate rarefaction waves,

$$
\left|\sigma_{i}\left(\eta\left(v_{i+1}(x(t))\right)-\eta\left(v_{i}(x(t))\right)\right)-\left(q\left(v_{i+1}(x(t))\right)-q\left(v_{i}(x(t))\right)\right)\right| \leqq C h^{\frac{3}{2}-2 \beta}
$$

for any $C^{2}$ weak entropy-entropy flux pair $(\eta, q)$ and the mechanical energyenergy flux $\left(\eta_{*}, q_{*}\right)$.

Finally, we define the approximate solution $v^{h}(x, t)=\left(\rho^{h}(x, t), \bar{m}^{h}(x, t)\right)$ of (4.1) in the strip $n \Delta t \leqq t<(n+1) \Delta t$ by the fractional step procedure:

$$
v^{h}(x, t)=v_{0}^{h}(x, t)+G\left(v_{0}^{h}(x, t), x, t\right)(t-n \Delta t) .
$$

\section{Spherically Symmetric Solutions and Nozzle Solutions}

Consider the spherically symmetric solutions of (1.1) in $\mathbf{R}^{N}$ :

$$
(\rho(\vec{x}, t), \vec{m}(\vec{x}, t), \phi(\vec{x}, t))=\left(\rho(x, t), m(x, t) \frac{\vec{x}}{x}, \phi(x, t)\right),
$$

where $x=|\vec{x}|, m(x, t)=\rho(x, t) u(x, t)$. Then (1.1)-(1.2) becomes

$$
\left\{\begin{array}{l}
v_{t}+f(v)_{x}=a(x) g(v)+G(v, x, t), \quad 1<x<2, t>0, \\
\left.v\right|_{t=0}=v_{0}(x) \\
\left.m\right|_{x=1}=\left.m\right|_{x=2}=0
\end{array}\right.
$$

where

$$
\begin{aligned}
v & =(\rho, m)^{\top}, \quad f(v)=\left(m, \frac{m^{2}}{\rho}+p(\rho)\right)^{\top}, \\
g(v) & =\left(m, \frac{m^{2}}{\rho}\right)^{\top}, \quad G=\left(0, G_{2}\right)^{\top},
\end{aligned}
$$

and $a(x)=-\frac{N-1}{x}=-\frac{A^{\prime}(x)}{A(x)}, A(x)=e^{-\int^{x} a(y) d y}=N \omega_{N} x^{N-1}, \omega_{N}=\frac{2 \pi^{N / 2}}{N \Gamma(N / 2)}$, with

$$
G_{2}(\rho, m, x, t)=\rho x^{1-N}\left(\int_{1}^{x}(\rho-D(\xi)) \xi^{N-1} d \xi+c(\rho, t)\right)-\frac{m}{\tau}
$$


where

$$
c(\rho, t)=c_{0}\left(\phi_{2}(t)-\phi_{1}(t)-\int_{1}^{2} s^{1-N} \int_{1}^{s}(\rho-D(\xi)) \xi^{N-1} d \xi d s\right)
$$

and $c_{0}=\frac{1}{\int_{1}^{2} s^{1-N} d s}$.

We construct the approximate solutions of (5.1) as the construction for (4.1) with $\bar{m}=m$ and, in this case, (4.7) becomes

$$
\left\{\begin{array}{l}
\rho^{h}(x, t)=\rho_{0}^{h}(x, t), \\
m^{h}(x, t)=m_{0}^{h}(x, t)+G_{2}\left(\rho_{0}^{h}(x, t), m_{0}^{h}(x, t), x, t\right)(t-n \Delta t),
\end{array}\right.
$$

for $n \Delta t \leqq t<(n+1) \Delta t$.

5.1. Uniform Estimates. In this section, we will make the $L^{\infty}$ estimates to get the uniform bounds of the approximate solution $v^{h}(x, t)=\left(\rho^{h}(x, t), m^{h}(x, t)\right)$. For simplicity of notation, we denote $C>0$, a universal constant depending only on $T$, throughout this paper.

First we have the following lemma about the conservation of particles.

Lemma 5.1. There exists a constant $C>0$, which depends only on the bounds of $A(x)$ and $\rho_{0}+\left|\frac{m_{0}}{\rho_{0}}\right|$, such that, for any $t \in[0, T]$,

$$
\int_{1}^{2} \rho_{0}^{h}(x, t) d x \leqq C+C \max _{j}\left\{\rho_{j}^{n}\right\} h^{\beta},
$$

for some $n$ with $t \in[n \Delta t,(n+1) \Delta t)$.

Proof. By the construction of the approximate solutions, we have

$$
\begin{aligned}
& \int_{1}^{2} A(x) \rho_{0}^{h}(x,(n+1) \Delta t-0) d x+\int_{n \Delta t}^{(n+1) \Delta t} A(x(t)) \sum\left(\sigma\left[\rho_{0}^{h}\right]-\left[m_{0}^{h}\right]\right) d t \\
& =\int_{1}^{2} A(x) \rho_{0}^{h}(x, n \Delta t+0) d x+O\left(h^{\beta} \Delta t\right) .
\end{aligned}
$$

Using the Rankine-Hugoniot condition, one has

and then

$$
\int_{n \Delta t}^{(n+1) \Delta t} A(x(t)) \sum\left(\sigma\left[\rho_{0}^{h}\right]-\left[m_{0}^{h}\right]\right) d t=0
$$

$$
\begin{aligned}
\int_{1}^{2} A(x) \rho_{0}^{h}(x,(n+1) \Delta t-0) d x= & \int_{1}^{2} A(x)\left(\rho_{0}^{h}(x, n \Delta t+0)-\rho_{0}^{h}(x, n \Delta t-0)\right) d x \\
& +\int_{1}^{2} A(x) \rho_{0}^{h}(x, n \Delta t-0) d x+O\left(h^{\beta+1}\right) .
\end{aligned}
$$

By the construction and Lemma 3.2, we have

$$
\frac{1}{h} \int_{1+\left(j-\frac{1}{2}\right) h}^{1+\left(j+\frac{1}{2}\right) h} A(x)\left(\rho_{0}^{h}(x, n \Delta t-0)-\rho_{j}^{n}\right) d x=0,
$$


and

$$
\frac{1}{h} \int_{1+\left(j-\frac{1}{2}\right) h}^{1+\left(j+\frac{1}{2}\right) h} A(x)\left(\rho_{0}^{h}(x, n \Delta t+0)-\rho_{j}^{n}\right) d x=\rho_{j}^{n} O\left(h^{1+\beta}\right) .
$$

Then, by subtracting and adding the term $\rho_{j}^{n}$, we get

$$
\int_{1}^{2} A(x)\left(\rho_{0}^{h}(x, n \Delta t+0)-\rho_{0}^{h}(x, n \Delta t-0)\right) d x=\sum_{j} \rho_{j}^{n} O\left(h^{2+\beta}\right),
$$

and

$$
\begin{aligned}
& \int_{1}^{2} A(x) \rho_{0}^{h}(x,(n+1) \Delta t-0) d x \\
& \quad=\int_{1}^{2} A(x) \rho_{0}^{h}(x, n \Delta t-0) d x+\sum_{j} \rho_{j}^{n} O\left(h^{2+\beta}\right)+O\left(h^{1+\beta}\right) .
\end{aligned}
$$

Therefore, by induction on $n$, we have

$$
\int_{1}^{2} A(x) \rho_{0}^{h}(x, n \Delta t-0) d x=\int_{1}^{2} A(x) \rho_{0}^{h}(x, 0) d x+\sum_{j} \rho_{j}^{n} O\left(h^{1+\beta}\right)+O\left(h^{\beta}\right), \quad \forall n .
$$

For any $t \in[0, T], t \in[n \Delta t,(n+1) \Delta t)$ for some $n$, then by the construction of the approximate solutions, the Rankine-Hugoniot condition, and the above estimates, one has

$$
\int_{1}^{2} A(x) \rho_{0}^{h}(x, t) d x=\int_{1}^{2} A(x) \rho_{0}(x) d x+\sum_{j} \rho_{j}^{n} O\left(h^{1+\beta}\right)+O\left(h^{\beta}\right) .
$$

Since $A(x) \geqq N \omega_{N}$ for any $x \in(1,2)$, Lemma 5.1 follows from (5.6). The proof is completed.

Let $\Pi_{T}=[1,2] \times[0, T]$. We have the following uniform estimate.

Theorem 5.1. Suppose there exists a constant $C>0$ such that $0<\rho_{0}(x) \leqq C$ and $\left|u_{0}(x)\right| \leqq C$ for all $x \in(1,2)$. Then, for $h \leqq h_{0}$, there exists a positive constant $C(T)$, independent of $h$ and $\tau$, such that

$$
h^{\beta} \leqq \rho^{h}(x, t) \leqq C(T), \quad\left|u^{h}(x, t)\right| \leqq C(T), \quad(x, t) \in \Pi_{T} .
$$

Proof. Suppose, for small $h$,

Then Lemma 5.1 implies

$$
\sup _{(x, t)} \rho^{h}(x, t) \leqq \frac{1}{h^{\beta}}
$$

$$
\int_{1}^{2} \rho_{0}^{h}(x, t) d x \leqq C, \quad \forall t \in[0, T] .
$$

By the construction of $\left(\rho^{h}, m^{h}\right)$, we have $\rho^{h}(x, t) \geqq h^{\beta}$, for $(x, t) \in \Pi_{T}$. By $(5.3)$ and (5.7),

$$
\left|c\left(\rho_{0}^{h}, t\right)\right| \leqq c_{0}\left(\left|\phi_{2}(t)-\phi_{1}(t)\right|+\int_{1}^{2} \rho_{0}^{h}(\xi, t) d \xi+\int_{1}^{2}|D(\xi)| d \xi\right) \leqq C .
$$


In $n \Delta t \leqq t<(n+1) \Delta t$, we estimate the Riemann invariant $w$ using (5.4) and (5.7):

$$
\begin{aligned}
w\left(v^{h}(x, t)\right)= & w\left(v_{0}^{h}(x, t)\right) \\
& +\left(x^{1-N}\left(\int_{1}^{x}\left(\rho_{0}^{h}-D(\xi)\right) \xi^{N-1} d \xi+c\left(\rho_{0}^{h}, t\right)\right)-\frac{w_{0}^{h}+z_{0}^{h}}{2 \tau}\right)(t-n \Delta t) \\
\leqq & w\left(v_{0}^{h}(x, t)\right)\left(1-\frac{t-n \Delta t}{2 \tau}\right)-\frac{z\left(v_{0}^{h}(x, t)\right)}{2 \tau}(t-n \Delta t)+C \Delta t .
\end{aligned}
$$

By Lemma 3.1 and the construction of $\left(\rho^{h}, m^{h}\right)$, we have

$$
\left\{\begin{array}{l}
w\left(v_{0}^{h}(x, t)\right) \leqq \max \left(\sup _{x} w\left(v_{0}^{h}(x, n \Delta t+0)\right), 1\right)(1+C \Delta t), \\
z\left(v_{0}^{h}(x, t)\right) \geqq \min \left(\inf _{x} z\left(v_{0}^{h}(x, n \Delta t+0)\right),-1\right)(1+C \Delta t),
\end{array}\right.
$$

for $h \leqq h_{0}$. Then

$$
\begin{aligned}
w\left(v^{h}(x, t)\right) \leqq & \max \left(\sup _{x} w\left(v_{0}^{h}(x, n \Delta t+0)\right), 1\right)(1+C \Delta t)\left(1-\frac{t-n \Delta t}{2 \tau}\right) \\
& -\min \left(\inf _{x} z\left(v_{0}^{h}(x, n \Delta t+0)\right),-1\right)(1+C \Delta t) \frac{t-n \Delta t}{2 \tau}+C \Delta t .
\end{aligned}
$$

Similarly, we have

$$
\begin{aligned}
z\left(v^{h}(x, t)\right) \geqq & \min \left(\inf _{x} z\left(v_{0}^{h}(x, n \Delta t+0)\right),-1\right)(1+C \Delta t)\left(1-\frac{t-n \Delta t}{2 \tau}\right) \\
& -\max \left(\sup _{x} w\left(v_{0}^{h}(x, n \Delta t+0)\right), 1\right)(1+C \Delta t) \frac{t-n \Delta t}{2 \tau}+C \Delta t .
\end{aligned}
$$

Note that

$$
\rho_{j}^{n}=\frac{1}{h} \int_{1+\left(j-\frac{1}{2}\right) h}^{1+\left(j+\frac{1}{2}\right) h} \rho^{h}(x, n \Delta t-0) d x(1+O(h)),
$$

where the bound $O(h)$ depends only on the bound of $A(x)$. Then

$$
w\left(v_{j}^{n}\right)=w\left(\bar{v}_{j}^{n}\right)(1+O(h)), \quad z\left(v_{j}^{n}\right)=z\left(\bar{v}_{j}^{n}\right)(1+O(h)),
$$

where $\bar{v}_{j}^{n}=\frac{1}{h} \int_{1+\left(j-\frac{1}{2}\right) h}^{1+\left(j+\frac{1}{2}\right) h} v^{h}(x, n \Delta t-0) d x$.

Set $M_{n}=\max \left(\sup _{x} w\left(v_{0}^{h}(x, n \Delta t+0)\right),-\inf _{x} z\left(v_{0}^{h}(x, n \Delta t+0)\right), 1\right)$. Then

Thus

$$
M_{n+1} \leqq M_{n}(1+C \Delta t)+C \Delta t .
$$

$$
M_{n+1} \leqq M_{0}(1+C \Delta t)^{\frac{T}{\Delta t}+1}+C T(1+C \Delta t)^{\frac{T}{\Delta t}} \leqq C(T) .
$$

This implies

$$
\left\{\begin{array}{l}
w\left(v^{h}(x, t)\right) \leqq C(T)(1+C \Delta t)+C \Delta t \leqq C(T), \\
-z\left(v^{h}(x, t)\right) \leqq C(T)(1+C \Delta t)+C \Delta t \leqq C(T), \\
w\left(v^{h}(x, t)\right)-z\left(v^{h}(x, t)\right) \geqq \frac{2 h^{\beta \theta}}{\theta} .
\end{array}\right.
$$


Therefore, there exists a constant $C(T)>0$, which is independent of $h$ and $\tau$, such that

$$
h^{\beta} \leqq \rho^{h}(x, t) \leqq C(T), \quad\left|u^{h}(x, t)\right|=\left|\frac{m^{h}(x, t)}{\rho^{h}(x, t)}\right| \leqq C(T) .
$$

Choose $h_{0}>0$ such that $h_{0}^{\beta}<\frac{1}{C(T)}$. Then, for $h \leqq h_{0}$,

$$
h^{\beta} \leqq \rho^{h}(x, t) \leqq C(T)<\frac{1}{h^{\beta}} .
$$

This completes the proof.

5.2. $H^{-1}$ Compactness of Entropy Measures. We need the following basic lemma (cf. $[7,43])$ to prove the $H^{-1}$ compactness of entropy measures for the approximate solutions $\left(\rho^{h}, m^{h}\right)$.

Lemma 5.2. Let $\Omega \subset \mathbf{R}^{N}$ be a bounded domain, then

(compact set of $\left.W^{-1, q}(\Omega)\right) \cap\left(\right.$ bounded set of $\left.W^{-1, r}(\Omega)\right)$

$$
\subset\left(\text { compact set of } W_{\mathrm{loc}}^{-1,2}(\Omega)\right),
$$

where $q$ and $r$ are constants, $1<q \leqq 2<r<\infty$.

Theorem 5.2. If $\left\{v^{h}\right\}$ are the approximate solutions, then the measure sequence

$$
\eta\left(v^{h}\right)_{t}+q\left(v^{h}\right)_{x}
$$

is a compact subset of $H_{\mathrm{loc}}^{-1}(\Omega)$ for all weak entropy pairs $(\eta, q)$, where $\Omega$ is any bounded and open set in $\Pi_{T}$.

Proof. For any $\psi \in C^{1}\left(\Pi_{T}\right)$, we have

$$
\begin{aligned}
& \iint_{\Pi_{T}}\left(\eta\left(v^{h}\right) \psi_{t}+q\left(v^{h}\right) \psi_{x}\right) d x d t \\
& \quad=A(\psi)+M(\psi)+N(\psi)+L_{1}(\psi)+L_{2}(\psi)+\Sigma(\psi)+E(\psi),
\end{aligned}
$$

where

$$
\begin{aligned}
A(\psi) & =\iint_{\Pi_{T}}\left(\left(\eta\left(v^{h}\right)-\eta\left(v_{0}^{h}\right)\right) \psi_{t}+\left(q\left(v^{h}\right)-q\left(v_{0}^{h}\right)\right) \psi_{x}\right) d x d t \\
M(\psi) & =\int_{1}^{2} \psi(x, T) \eta\left(v_{0}^{h}(x, T)\right) d x-\int_{1}^{2} \psi(x, 0) \eta\left(v_{0}^{h}(x, 0)\right) d x \\
N(\psi) & =-\int_{\Pi_{T}} a(x) g\left(v_{0}^{h}\right) \nabla \eta\left(v_{0}^{h}\right) \psi(x, t) d x d t \\
L_{1}(\psi) & =\sum_{j, n} \psi_{j}^{n} \int_{1+\left(j-\frac{1}{2}\right) h}^{1+\left(j+\frac{1}{2}\right) h}\left(\eta\left(v_{0-}^{n}\right)-\eta\left(v_{0+}^{n}\right)\right) d x \\
L_{2}(\psi) & =\sum_{j, n} \int_{1+\left(j-\frac{1}{2}\right) h}^{1+\left(j+\frac{1}{2}\right) h}\left(\eta\left(v_{0-}^{n}\right)-\eta\left(v_{0+}^{n}\right)\right)\left(\psi-\psi_{j}^{n}\right) d x
\end{aligned}
$$


and

$$
\Sigma(\psi)=\int_{0}^{T} \sum(\sigma[\eta]-[q]) \psi(x(t), t) d t, \quad|E(\psi)| \leqq C h^{\beta}\|\psi\|_{H^{1}},
$$

where $v_{0 \pm}^{n}=v_{0}^{h}(x, n \Delta t \pm 0), \psi_{j}^{n}=\psi(1+j h, n \Delta t)$, the summation in $\Sigma(\psi)$ is taken over all discontinuities in $v_{0}^{h}$ at a fixed time $t, \sigma$ is the propagating speed of the discontinuity, and $E(\psi)$ is the error term including the error in the steady-state solutions and the error near the vacuum in the construction of approximate solutions, and

$$
\begin{aligned}
& {[\eta]=\eta\left(v_{0}^{h}(x(t)+0, t)\right)-\eta\left(v_{0}^{h}(x(t)-0, t)\right),} \\
& {[q]=q\left(v_{0}^{h}(x(t)+0, t)\right)-q\left(v_{0}^{h}(x(t)-0, t)\right),}
\end{aligned}
$$

are the jump of $\eta\left(v_{0}^{h}(x, t)\right)$ and $q\left(v_{0}^{h}(x, t)\right)$ across a discontinuity $S=(x(t), t)$ in $v_{0}^{h}(x, t)$.

First we have the following two lemmas.

Lemma 5.3. For any $n$ and $h \leqq h_{0}$,

$$
\frac{1}{h} \int_{1+\left(j-\frac{1}{2}\right) h}^{1+\left(j+\frac{1}{2}\right) h}\left(\rho^{h}(x, n \Delta t-0)-\rho_{j}^{n}\right) d x=O(h) .
$$

This directly follows from (5.5) and

$$
\begin{aligned}
& A(1+j h) \frac{1}{h} \int_{1+\left(j-\frac{1}{2}\right) h}^{1+\left(j+\frac{1}{2}\right) h}\left(\rho^{h}(x, n \Delta t-0)-\rho_{j}^{n}\right) d x \\
& =\frac{1}{h} \int_{1+\left(j-\frac{1}{2}\right) h}^{1+\left(j+\frac{1}{2}\right) h}(A(1+j h)-A(x))\left(\rho^{h}(x, n \Delta t-0)-\rho_{j}^{n}\right) d x=O(h) .
\end{aligned}
$$

Lemma 5.4. There exists a constant $C>0$ such that

(1) $\sum_{j, n} \int_{1+\left(j-\frac{1}{2}\right) h}^{1+\left(j+\frac{1}{2}\right) h} \int_{0}^{1}(1-s)\left(v_{0 \pm}^{n}-v_{j}^{n}\right)^{\top} \nabla^{2} \eta_{*}\left(v_{j}^{n}+s\left(v_{0 \pm}^{n}-v_{j}^{n}\right)\right)\left(v_{0 \pm}^{n}-v_{j}^{n}\right) d s d x \leqq C$,

(2) $\sum_{j, n} \int_{1+\left(j-\frac{1}{2}\right) h}^{1+\left(j+\frac{1}{2}\right) h}\left|v_{0 \pm}^{n}-v_{j}^{n}\right|^{2} d x \leqq C$.

Proof. We substitute $(\eta, q)=\left(\eta_{*}^{h}, q_{*}^{h}\right)$ :

$$
\begin{aligned}
\eta_{*}^{h} & =\eta_{*}(v)-\eta_{*}(\bar{v})-\nabla \eta_{*}(\bar{v})(v-\bar{v}), \\
q_{*}^{h} & =q_{*}(v)-q_{*}(\bar{v})-\nabla \eta_{*}(\bar{v})(f(v)-f(\bar{v})),
\end{aligned}
$$

with $\bar{v}=\left(h^{\beta}, 0\right)$, and $\psi \equiv 1$ in the equality (5.8) to obtain

$$
\sum_{j, n} \int_{1+\left(j-\frac{1}{2}\right) h}^{1+\left(j+\frac{1}{2}\right) h}\left(\eta_{*}^{h}\left(v_{0-}^{n}\right)-\eta_{*}^{h}\left(v_{0+}^{n}\right)\right) d x+\int_{0}^{T} \sum\left(\sigma\left[\eta_{*}^{h}\right]-\left[q_{*}^{h}\right]\right) d t \leqq C_{0}
$$


Rewrite the first term as

$$
\begin{aligned}
& \sum_{j, n} \int_{1+\left(j-\frac{1}{2}\right) h}^{1+\left(j+\frac{1}{2}\right) h}\left(\eta_{*}^{h}\left(v_{0-}^{n}\right)-\eta_{*}^{h}\left(v_{0+}^{n}\right)\right) d x \\
& \quad=\sum_{j, n} \int_{1+\left(j-\frac{1}{2}\right) h}^{1+\left(j+\frac{1}{2}\right) h}\left(\eta_{*}^{h}\left(v_{0-}^{n}\right)-\eta_{*}^{h}\left(v_{j}^{n}\right)\right) d x-\sum_{j, n} \int_{1+\left(j-\frac{1}{2}\right) h}^{1+\left(j+\frac{1}{2}\right) h}\left(\eta_{*}^{h}\left(v_{0+}^{n}\right)-\eta_{*}^{h}\left(v_{j}^{n}\right)\right) d x .
\end{aligned}
$$

Then we have from the Taylor expansion, the construction of $v^{h}$, and Lemma 5.3 that

$$
\begin{aligned}
& \sum_{j, n} \int_{1+\left(j-\frac{1}{2}\right) h}^{1+\left(j+\frac{1}{2}\right) h}\left(\eta_{*}^{h}\left(v_{0-}^{n}\right)-\eta_{*}^{h}\left(v_{j}^{n}\right)\right) d x \\
& \quad=\nabla \eta_{*}^{h}\left(v_{j}^{n}\right) \sum_{j, n} \int_{1+\left(j-\frac{1}{2}\right) h}^{1+\left(j+\frac{1}{2}\right) h}\left(v_{0-}^{n}-v^{h}(x, n \Delta t-0)\right) d x+O(1) \\
& \quad+\sum_{j, n} \int_{1+\left(j-\frac{1}{2}\right) h}^{1+\left(j+\frac{1}{2}\right) h} \int_{0}^{1}(1-s)\left(v_{0-}^{n}-v_{j}^{n}\right)^{\top} \nabla^{2} \eta_{*}\left(v_{j}^{n}+s\left(v_{0-}^{n}-v_{j}^{n}\right)\right)\left(v_{0-}^{n}-v_{j}^{n}\right) d s d x,
\end{aligned}
$$

and

$$
\left|\nabla \eta_{*}^{h}\left(v_{j}^{n}\right) \sum_{j, n} \int_{1+\left(j-\frac{1}{2}\right) h}^{1+\left(j+\frac{1}{2}\right) h}\left(v_{0-}^{n}-v^{h}(x, n \Delta t-0)\right) d x\right| \leqq\left\|\nabla \eta_{*}^{h}\right\|_{\infty} \sum_{j, n} h\left\|G_{2}\right\|_{\infty} \Delta t \leqq C,
$$

and

$$
\begin{aligned}
& \sum_{j, n} \int_{1+\left(j-\frac{1}{2}\right) h}^{1+\left(j+\frac{1}{2}\right) h}\left(\eta_{*}^{h}\left(v_{0+}^{n}\right)-\eta_{*}^{h}\left(v_{j}^{n}\right)\right) d x=O\left(h^{1-2 \beta}\right) \\
& \quad+\sum_{j, n} \int_{1+\left(j-\frac{1}{2}\right) h}^{1+\left(j+\frac{1}{2}\right) h} \int_{0}^{1}(1-s)\left(v_{0+}^{n}-v_{j}^{n}\right)^{\top} \nabla^{2} \eta_{*}^{h}\left(v_{j}^{n}+s\left(v_{0+}^{n}-v_{j}^{n}\right)\right)\left(v_{0+}^{n}-v_{j}^{n}\right) d s d x \\
& \quad \leqq O\left(h^{1-2 \beta}\right)+C \sum_{j, n} h \frac{\left(\left|\rho_{j}^{n}\right|^{2}+\left|m_{j}^{n}\right|^{2}\right) h}{\rho_{j}^{n}-\rho_{j}^{n} O(\sqrt{h})} \leqq C .
\end{aligned}
$$

Here we actually proved the ${ }^{\prime}+{ }^{\prime}$ part of (1).

We have

$$
\begin{aligned}
& \sum_{j, n} \int_{1+\left(j-\frac{1}{2}\right) h}^{1+\left(j+\frac{1}{2}\right) h} \int_{0}^{1}(1-s)\left(v_{0-}^{n}-v_{j}^{n}\right)^{\top} \nabla^{2} \eta_{*}\left(v_{j}^{n}+s\left(v_{0+}^{n}-v_{j}^{n}\right)\right)\left(v_{0-}^{n}-v_{j}^{n}\right) d s d x \\
& \quad+\int_{0}^{T} \sum\left(\sigma\left[\eta_{*}^{h}\right]-\left[q_{*}^{h}\right]\right) d t \leqq C,
\end{aligned}
$$

from the above estimates and (5.9). 
Since $\sigma\left[\eta_{*}^{h}\right]-\left[q_{*}^{h}\right] \geqq-C h^{\frac{3}{2}-2 \beta}$, then

$$
\int_{0}^{T} \sum\left(\sigma\left[\eta_{*}^{h}\right]-\left[q_{*}^{h}\right]\right) d t \geqq-C T^{\frac{1}{2}-2 \beta} .
$$

Therefore, there exists a constant $C>0$ such that

$$
\sum_{j, n} \int_{1+\left(j-\frac{1}{2}\right) h}^{1+\left(j+\frac{1}{2}\right) h} \int_{0}^{1}(1-s)\left(v_{0-}^{n}-v_{j}^{n}\right)^{\top} \nabla^{2} \eta_{*}\left(v_{j}^{n}+s\left(v_{0_{-}}^{n}-v_{j}^{n}\right)\right)\left(v_{0_{-}}^{n}-v_{j}^{n}\right) d s d x \leqq C,
$$

and

$$
\int_{0}^{T} \sum\left(\sigma\left[\eta_{*}^{h}\right]-\left[q_{*}^{h}\right]\right) d t \leqq C
$$

This proves the ' - ' part of (1).

Since $\nabla^{2} \eta_{*}^{h}(r, r) \geqq c_{0}(r, r), c_{0}>0$, then (1) implies (2).

We now continue the proof of Theorem 5.2.

(a) From the lemmas in Sects. 2 and 3, Lemma 5.3, and Lemma 5.4,

$$
\begin{aligned}
& |M(\psi)| \leqq C\|\psi\|_{C_{0}(\Omega)}, \quad|N(\psi)| \leqq C\|\psi\|_{C_{0}(\Omega)} \\
& |\Sigma(\psi)| \leqq\|\psi\|_{C_{0}(\Omega)} \int_{0}^{T}\left(\sum\left(\sigma\left[\eta_{*}\right]-\left[q_{*}\right]\right)+h^{2(1-\beta)}\right) d t \leqq C\|\psi\|_{C_{0}(\Omega)},
\end{aligned}
$$

and

$$
\begin{aligned}
\left|L_{1}(\psi)\right| \leqq & \sum_{j, n} \psi_{j}^{n} \int_{1+\left(j-\frac{1}{2}\right) h}^{1+\left(j+\frac{1}{2}\right) h}\left(\eta\left(v_{0-}^{n}\right)-\eta\left(v_{j}^{n}\right)\right) d x|+| \sum_{j, n} \psi_{j}^{n} \int_{1+\left(j-\frac{1}{2}\right) h}^{1+\left(j+\frac{1}{2}\right) h}\left(\eta\left(v_{0+}^{n}\right)-\eta\left(v_{j}^{n}\right)\right) d x \mid \\
\leqq & C\|\psi\|_{C_{0}} \sum_{j, n} \int_{1+\left(j-\frac{1}{2}\right) h}^{1+\left(j+\frac{1}{2}\right) h} \int_{0}^{1}(1-s)\left(v_{0-}^{n}-v_{j}^{n}\right)^{\top} \\
& \times \nabla^{2} \eta_{*}\left(v_{j}^{n}+s\left(v_{0-}-v_{j}^{n}\right)\right)\left(v_{0-}^{n}-v_{j}^{n}\right) d s d x \\
& +C\|\psi\|_{C_{0}} \sum_{j, n} \int_{1+\left(j-\frac{1}{2}\right) h}^{1+\left(j+\frac{1}{2}\right) h} \int_{0}^{1}(1-s)\left(v_{0+}^{n}-v_{j}^{n}\right)^{\top} \\
& \times \nabla^{2} \eta_{*}\left(v_{j}^{n}+s\left(v_{0+}^{n}-v_{j}^{n}\right)\right)\left(v_{0+}^{n}-v_{j}^{n}\right) d s d x \\
& +\|\psi\|_{C_{0}}\left(O(1)+O\left(h^{1-2 \beta}\right)\right) \leqq C\|\psi\|_{C_{0}} .
\end{aligned}
$$

Hence

$$
\left|\left(M+N+L_{1}+\Sigma\right)(\psi)\right| \leqq C\|\psi\|_{C_{0}}
$$

that is

$$
\left\|M+N+L_{1}+\Sigma\right\|_{C_{0}^{*}} \leqq C .
$$

By the embedding theorem, $\left(C_{0}(\Omega)\right)^{*} \hookrightarrow W^{-1, q_{1}}$, for $1<q_{1}<2, M+N+L_{1}+\Sigma$ is compact in $W^{-1, q_{1}}(\Omega)$. 
(b) For any $\psi \in C_{0}^{\alpha}(\Omega), \frac{1}{2}<\alpha<1$, using Hölder's inequality, we have

$$
\begin{aligned}
& \left|L_{2}(\psi)\right| \leqq \sum_{j, n} \int_{1+\left(j-\frac{1}{2}\right) h}^{1+\left(j+\frac{1}{2}\right) h}\left|\psi-\psi_{j}^{n}\right|\left(\left|\eta\left(v_{0-}^{n}\right)-\eta\left(v_{j}^{n}\right)\right|+\left|\eta\left(v_{0+}^{n}\right)-\eta\left(v_{j}^{n}\right)\right|\right) d x \\
& \leqq C\|\psi\|_{C_{0}^{\alpha}} h^{\alpha-\frac{1}{2}}\|\nabla \eta\|_{\infty}\left(\sum_{j, n} \int_{1+\left(j-\frac{1}{2}\right) h}^{1+\left(j+\frac{1}{2}\right) h}\left|v_{0-}^{n}-v_{j}^{n}\right|^{2} d x\right. \\
& \left.+\sum_{j, n} \int_{1+\left(j-\frac{1}{2}\right) h}^{1+\left(j+\frac{1}{2}\right) h}\left|v_{0+}^{n}-v_{j}^{n}\right|^{2} d x\right)^{\frac{1}{2}} \\
& \leqq C h^{\alpha-\frac{1}{2}}\|\psi\|_{C_{0}^{\alpha}} \text {. }
\end{aligned}
$$

By the Sobolev theorem: $W_{0}^{1, p}(\Omega) \subset C_{0}^{\alpha}(\Omega), 0<\alpha<1-\frac{2}{p}$, we have

$$
\left|L_{2}(\psi)\right| \leqq C h^{\alpha-\frac{1}{2}}\|\psi\|_{W_{0}^{1, p}(\Omega)}, \quad p>\frac{2}{1-\alpha}
$$

that is,

$$
\left\|L_{2}\right\|_{W^{-1, q_{2(\Omega)}}} \leqq C h^{\alpha-\frac{1}{2}} \rightarrow 0, \quad h \rightarrow 0,
$$

for $1<q_{2}<\frac{2}{1+\alpha}$. Therefore $L_{2}$ is compact in $W^{-1, q_{2}}$, and then $M+N+L+\Sigma$ is compact in $W^{-1, q_{0}}$, where $1<q_{0}=\min \left(q_{1}, q_{2}\right)<\frac{2}{1+\alpha}$.

The uniform boundedness of the approximate solutions implies $M+N+L+\Sigma$ is bounded in $W^{-1, r}, r>1$. By Lemma $5.2, M+N+L+\Sigma$ is compact in $H_{\mathrm{loc}}^{-1}$.

(c) Finally, for $A(\psi)$ we have

$$
\|A(\psi)\| \leqq \iint_{\Pi_{T}}\left(\|\nabla \eta\|_{\infty}+\|\nabla q\|_{\infty}\right)\left(\left|\psi_{t}\right|+\left|\psi_{x}\right|\right)\left|v^{h}-v_{0}^{h}\right| d x d t \leqq C h\|\psi\|_{H_{0}^{1}(\Omega)} .
$$

Since $C_{0}^{\infty}(\Omega)$ is dense in $H_{0}^{1}(\Omega)$, then

$$
\|A\|_{H_{\mathrm{loc}}^{-1}(\Omega)} \leqq C h \rightarrow 0, \quad \text { as } h \rightarrow 0,
$$

so $A$ is compact in $H_{\mathrm{loc}}^{-1}(\Omega)$.

We know from $\|E\|_{H^{-1}} \leqq C h^{\beta} \rightarrow 0$, as $h \rightarrow 0$, that $E$ is compact in $H_{\text {loc }}^{-1}(\Omega)$. Therefore $A+M+N+L+\Sigma+E$ is compact in $H_{\mathrm{loc}}^{-1}(\Omega)$, which means that $\eta\left(v^{h}\right)_{t}$ $+q\left(v^{h}\right)_{x}$ is compact in $H_{\text {loc }}^{-1}(\Omega)$.

5.3. Convergence and Existence. In this section, we prove that (5.1) has a weak entropy solution, which is the limit function of the approximate solutions.

Definition 5.1. A measurable function $v(x, t)=(\rho(x, t), m(x, t))$ is a weak entropy solution of (5.1) if, for any test function $\psi \in C_{0}^{1}\left(\Pi_{T}\right)$ with $\psi(1, t)=\psi(2, t)=$ $\psi(x, T)=0$,

$\iint_{\Pi_{T}}\left(v \psi_{t}+f(v) \psi_{x}+(a(x) g(v)+G(v, x, t)) \psi\right) d x d t+\int_{1}^{2} v_{0}(x) \psi(x, 0) d x=0$, 
and, along any shock discontinuity with left state $v_{-}$, right state $v_{+}$, and speed $\sigma$,

$$
\sigma\left(\eta\left(v_{+}\right)-\eta\left(v_{-}\right)\right)-\left(q\left(v_{+}\right)-q\left(v_{-}\right)\right) \geqq 0,
$$

for any convex weak entropy pair $(\eta, q)$. $[6,7])$ :

Now we introduce the following compensated compactness framework (see

Lemma 5.5. Assume that the approximate solutions $v^{h}=\left(\rho^{h}, m^{h}\right)$ satisfies

(1) There is a constant $C>0$ such that $0 \leqq \rho^{h}(x, t) \leqq C,\left|\frac{m^{h}(x, t)}{\rho^{h}(x, t)}\right| \leqq C$;

(2) The measure $\eta\left(v^{h}\right)_{t}+q\left(v^{h}\right)_{x}$ is compact in $H_{\text {loc }}^{-1}(\Omega)$, for all weak entropy pairs $(\eta, q)$, where $\Omega \subset \Pi_{T}$ is any bounded and open set.

Then, for $1<\gamma \leqq 5 / 3$, there exists a convergent subsequence (still labeled) $v^{h}$ such that $v^{h}(x, t) \rightarrow v(x, t)=(\rho(x, t), m(x, t))$ almost everywhere.

In Sects. 5.1 and 5.2, we have proved that the approximate solutions $v^{h}(x, t)$ constructed in Sect. 4 for (5.1) satisfies (1) and (2) of Lemma 5.5. Thus we have the following theorem.

Theorem 5.3. There is a convergent subsequence (still labeled) $v^{h}$ in the approximate solutions $v^{h}(x, t)=\left(\rho^{h}(x, t), m^{h}(x, t)\right)$ such that

$$
v^{h}(x, t) \rightarrow v(x, t)=(\rho(x, t), m(x, t)), \text { a.e. } \text { as } h \rightarrow 0,
$$

and that the function $v(x, t)$ is a weak entropy solution of (5.1) in the sense of Definition 5.1 and satisfies

$$
0 \leqq \rho(x, t) \leqq C(T), \quad\left|\frac{m(x, t)}{\rho(x, t)}\right| \leqq C(T),
$$

for $(x, t) \in \Pi_{T}$, where $C(T)>0$ is a constant.

Proof. The convergence follows from Lemma 5.5. Now we prove that $v(x, t)$ satisfies (5.10) and (5.11).

(a) Let $\psi \in C_{0}^{1}\left(\Pi_{T}\right)$ be any test function with $\psi(1, t)=\psi(2, t)=\psi(x, T)=0$ and set $\bar{\psi}(x, t)=\frac{\psi(x, t)}{A(x)} \in C_{0}^{1}\left(\Pi_{T}\right)$. One has

$$
\begin{aligned}
& \iint_{\Pi_{T}}\left(\rho^{h} \psi_{t}+m^{h} \psi_{x}+a(x) m^{h} \psi\right) d x d t+\int_{1}^{2} \rho_{0}^{h}(x) \psi(x, 0) d x \\
& =\iint_{\Pi_{T}}\left(A(x) \rho^{h} \bar{\psi}_{t}+A(x) m^{h} \bar{\psi}_{x}\right) d x d t+\int_{1}^{2} A(x) \rho_{0}^{h}(x) \bar{\psi}(x, 0) d x \\
& =\iint_{I_{T}} A(x)\left(m^{h}-m_{0}^{h}\right) \bar{\psi}_{x} d x d t+\int_{0}^{T} \sum A(x(t))\left(\sigma\left[\rho_{0}^{h}\right]-\left[m_{0}^{h}\right]\right) \bar{\psi}(x(t), t) d t \\
& \quad+I_{11}+I_{12}+O\left(h^{\beta}\right)\|\bar{\psi}\|_{H^{1}},
\end{aligned}
$$


where

$$
\begin{aligned}
& I_{11}=\sum_{j, n} \bar{\psi}_{j}^{n} \int_{1+\left(j-\frac{1}{2}\right) h}^{1+\left(j+\frac{1}{2}\right) h} A(x)\left(\rho_{0}^{h}(x, n \Delta t-0)-\rho_{0}^{h}(x, n \Delta t+0)\right) d x \\
& I_{12}=\sum_{j, n} \int_{1+\left(j-\frac{1}{2}\right) h}^{1+\left(j+\frac{1}{2}\right) h} A(x)\left(\bar{\psi}-\bar{\psi}_{j}^{n}\right)\left(\rho_{0}^{h}(x, n \Delta t-0)-\rho_{0}^{h}(x, n \Delta t+0)\right) d x .
\end{aligned}
$$

By the fractional step procedure and the Rankine-Hugoniot condition, one has

$$
\iint_{\Pi_{T}} A(x)\left(m^{h}-m_{0}^{h}\right) \bar{\psi}_{x} d x d t=O(h), \quad \int_{0}^{T} \sum A(x(t))\left(\sigma\left[\rho_{0}^{h}\right]-\left[m_{0}^{h}\right]\right) \bar{\psi}(x(t), t) d t=0 .
$$

By the construction of $v^{h}(x, t)$, Lemma 3.2 and Lemma 5.4, one has

$$
\begin{aligned}
\left|I_{11}\right| & =\left|\sum_{j, n} \bar{\psi}_{j}^{n} \int_{1+\left(j-\frac{1}{2}\right) h}^{1+\left(j+\frac{1}{2}\right) h}\left(A(x)\left(\rho_{0}^{h}(x, n \Delta t-0)-\rho_{j}^{n}\right)+A(x)\left(\rho_{j}^{n}-\rho_{0}^{h}(x, n \Delta t+0)\right)\right) d x\right| \\
& =\left|\sum_{j, n} \bar{\psi}_{j}^{n} h O\left(h^{1+\beta}\right)\right| \leqq O\left(h^{\beta}\right)\|\bar{\psi}\|_{C_{0}}, \\
\left|I_{12}\right| & \leqq C\|\bar{\psi}\|_{C_{0}^{1}} \sqrt{h}\left(\sum_{j, n} \int_{1+\left(j-\frac{1}{2}\right) h}^{1+\left(j+\frac{1}{2}\right) h}\left(\left|\rho_{0}^{h}(x, n \Delta t-0)-\rho_{j}^{n}\right|^{2}+\left|\rho_{j}^{n}-\rho_{0}^{h}(x, n \Delta t+0)\right|^{2}\right) d x\right)^{\frac{1}{2}} \\
& \leqq O(\sqrt{h})\|\bar{\psi}\|_{C_{0}^{1}} .
\end{aligned}
$$

Taking the limit $h \rightarrow 0$ in (5.12) and using the dominated convergence theorem, one has

$$
\iint_{\Pi_{T}}\left(\rho \psi_{t}+m \psi_{x}+a(x) m \psi\right) d x d t+\int_{1}^{2} \rho_{0}(x) \psi(x, 0) d x=0
$$

(b) Similarly to (a), by the fractional step procedure and the Rankine-Hugoniot condition,

$$
\begin{aligned}
& \iint_{\Pi_{T}}\left(m^{h} \psi_{t}+\left(\frac{\left(m^{h}\right)^{2}}{\rho^{h}}+p\left(\rho^{h}\right)\right) \psi_{x}+\left(a(x) \frac{\left(m^{h}\right)^{2}}{\rho^{h}}+G_{2}\left(v^{h}, x, t\right)\right) \psi\right) d x d t \\
& \quad+\int_{1}^{2} m_{0}^{h}(x) \psi(x, 0) d x=I_{21}+I_{22}+O(h)\|\psi\|_{C_{0}^{1}}+O\left(h^{\beta}\right)\|\psi\|_{H^{1}}
\end{aligned}
$$


where

$$
\begin{aligned}
I_{21}= & \sum_{j, n} \psi_{j}^{n} \int_{1+\left(j-\frac{1}{2}\right) h}^{1+\left(j+\frac{1}{2}\right) h}\left(m_{0}^{h}(x, n \Delta t-0)-m_{0}^{h}(x, n \Delta t+0)\right) d x \\
& +\iint_{\Pi_{T}} G_{2}\left(v_{0}^{h}, x, t\right) \psi d x d t, \\
I_{22}= & \sum_{j, n} \int_{1+\left(j-\frac{1}{2}\right) h}^{1+\left(j+\frac{1}{2}\right) h}\left(\psi-\psi_{j}^{n}\right)\left(m_{0}^{h}(x, n \Delta t-0)-m_{0}^{h}(x, n \Delta t+0)\right) d x .
\end{aligned}
$$

By the construction of $v^{h}$, Lemma 3.1, and a similar estimate in [16] (also see [35]),

$$
\begin{aligned}
\left|I_{21}\right| \leqq & \left|\sum_{j, n} \psi_{j}^{n} \int_{1+\left(j-\frac{1}{2}\right) h}^{1+\left(j+\frac{1}{2}\right) h}\left[\left(m^{h}(x, n \Delta t-0)-m_{j}^{n}\right)+\left(m_{j}^{n}-m_{0}^{h}(x, n \Delta t+0)\right)\right] d x\right| \\
& +\mid \sum_{j, n} \int_{(n-1) \Delta t}^{n \Delta t} \int_{1+\left(j-\frac{1}{2}\right) h}^{1+\left(j+\frac{1}{2}\right) h}\left(G_{2}\left(v_{0}^{h}(x, t), x, t\right) \psi(x, t)\right. \\
& \left.-G_{2}\left(v_{0}^{h}(x, n \Delta t-0), x, n \Delta t-0\right) \psi_{j}^{n}\right) d x d t \mid \\
\leqq & \|\psi\|_{C_{0}^{1}}\left(O(\sqrt{h})+O\left(h \delta^{-r_{1}}\right)+O\left(\delta^{r_{2}}\right)\right)
\end{aligned}
$$

where $\delta>0$ is an arbitrary and sufficiently small constant, $r_{1}, r_{2}>0$ are constants (cf. [16]).

By Lemma 5.4 and a similar estimate to $I_{12}$,

$$
\left|I_{22}\right| \leqq O(\sqrt{h})\|\psi\|_{C_{0}^{1}} .
$$

Taking the limit $h \rightarrow 0$ in (5.13) and noting that $\delta$ is arbitrarily small, we conclude

$$
\begin{aligned}
& \iint_{\Pi_{T}}\left(m \psi_{t}+\left(\frac{m^{2}}{\rho}+p(\rho)\right) \psi_{x}+\left(a(x) \frac{m^{2}}{\rho}+G_{2}(v, x, t)\right) \psi\right) d x d t \\
& \quad+\int_{1}^{2} m_{0}(x) \psi(x, 0) d x=0 .
\end{aligned}
$$

(c) For any convex weak entropy pair $(\eta, q)$ and any nonnegative test function $\psi \in C_{0}^{1}\left(\Pi_{T}\right)$ with $\psi(1, t)=\psi(2, t)=\psi(x, T)=0$,

$$
\begin{aligned}
& \iint_{\Pi_{T}}\left(\eta\left(v^{h}\right) \psi_{t}+q\left(v^{h}\right) \psi_{x}+\left(a(x) g\left(v^{h}\right)+G\left(v^{h}, x, t\right)\right) \nabla \eta\left(v^{h}\right) \psi\right) d x d t \\
& \quad+\int_{1}^{2} \eta\left(v_{0}^{h}(x)\right) \psi(x, 0) d x=A(\psi)+\tilde{A}(\psi)+\Sigma(\psi)+L_{1}(\psi)+L_{2}(\psi)+E(\psi),
\end{aligned}
$$

where the operators $A, \Sigma, L_{1}, L_{2}, E$ are the same as those in (5.8) and

$$
\tilde{A}(\psi)=\iint_{\Pi_{T}}\left(\left(a(x) g\left(v^{h}\right)+G\left(v^{h}, x, t\right)\right) \nabla \eta\left(v^{h}\right)-a(x) g\left(v_{0}^{h}\right) \nabla \eta\left(v_{0}^{h}\right)\right) \psi d x d t .
$$


As in Sect. 5.2, and since $\sigma[\eta]-[q] \geqq-C h^{\frac{3}{2}-2 \beta}$, we have

$$
\begin{aligned}
& A(\psi) \geqq-C h\|\psi\|_{H_{0}^{1}}, \quad \tilde{A}(\psi) \geqq-C\|\psi\|_{C_{0}}, \\
& \Sigma(\psi)=\int_{0}^{T} \sum(\sigma[\eta]-[q]) \psi(x(t), t) d t \geqq-C h^{\frac{1}{2}-2 \beta}\|\psi\|_{C_{0}} .
\end{aligned}
$$

Moreover, as in Sect. 5.2,

$$
\begin{aligned}
L_{1}(\psi)= & \sum_{j, n} \psi_{j}^{n} \int_{1+\left(j-\frac{1}{2}\right) h}^{1+\left(j+\frac{1}{2}\right) h} \int_{0}^{1}(1-s)\left(v_{0-}^{n}-v_{j}^{n}\right)^{\top} \\
& \times \nabla^{2} \eta\left(v_{j}^{n}+s\left(v_{0-}^{n}-v_{j}^{n}\right)\right)\left(v_{0-}^{n}-v_{j}^{n}\right) d s d x \\
& -\sum_{j, n} \psi_{j}^{n} \int_{1+\left(j-\frac{1}{2}\right) h}^{1+\left(j+\frac{1}{2}\right) h} \int_{0}^{1}(1-s)\left(v_{0+}^{n}-v_{j}^{n}\right)^{\top} \\
& \times \nabla^{2} \eta\left(v_{j}^{n}+s\left(v_{0+}^{n}-v_{j}^{n}\right)\right)\left(v_{0+}^{n}-v_{j}^{n}\right) d s d x \\
& +\sum_{j, n} \psi_{j}^{n} h\left(O(h)+O\left(h^{2-2 \beta}\right)\right) \geqq-C\|\psi\|_{C_{0}}\left(\left\|\rho \nabla^{2} \eta(v)\right\|_{\infty}+C\right), \\
\left|L_{2}(\psi)\right| \leqq & \sum_{j, n} \int_{1+\left(j-\frac{1}{2}\right) h}^{1+\left(j+\frac{1}{2}\right) h}\left|\psi(x, n \Delta t)-\psi_{j}^{n}\right|\left(\left|\eta\left(v_{0-}^{n}\right)-\eta\left(v_{j}^{n}\right)\right|+\left|\eta\left(v_{0+}^{n}\right)-\eta\left(v_{j}^{n}\right)\right|\right) d x \\
\leqq & C\|\psi\|_{C_{0}^{1}} \sqrt{h}\|\nabla \eta\|_{\infty}\left(\sum_{j, n} \int_{1+\left(j-\frac{1}{2}\right) h}^{1+\left(j+\frac{1}{2}\right) h}\left(\left|v_{0-}^{n}-v_{j}^{n}\right|^{2}+\left|v_{0+}^{n}-v_{j}^{n}\right|^{2}\right) d x\right)^{\frac{1}{2}} \\
\leqq & C\|\psi\|_{C_{0}^{1}} \sqrt{h} .
\end{aligned}
$$

Taking the limit $h \rightarrow 0$ in (5.14) and using the dominated convergence theorem, we verify that the limit function $v=(\rho, m)$ satisfies

$$
\eta(v)_{t}+q(v)_{x}-(a(x) g(v)+G(v, x, t)) \nabla \eta(v) \leqq C,
$$

in the sense of distributions. Using the standard procedure (cf. [30]), we conclude that the limit function $v(x, t)$ satisfies the entropy condition (5.11) along any shock wave. And the uniform boundedness of $v^{h}(x, t)$ implies the boundedness of the weak solution $v(x, t)$.

5.4. Nozzle Solutions. Now we consider the following equations for the nozzle flow:

$$
\left\{\begin{array}{l}
(A \rho)_{t}+(A m)_{x}=0, \\
(A m)_{t}+\left(A \frac{m^{2}}{\rho}\right)_{x}+A p(\rho)_{x}=A G_{2},
\end{array}\right.
$$


$1<x<2, t>0$, with initial-boundary conditions:

$$
\left\{\begin{array}{l}
\left.(\rho, m)\right|_{t=0}=\left(\rho_{0}(x), m_{0}(x)\right) \\
\left.m\right|_{x=1}=\left.m\right|_{x=2}=0
\end{array}\right.
$$

where $A(x) \in C^{2}$ is the cross-sectional area of the nozzle at $x$ and $G_{2}$ is the same as in (5.2).

Set $a(x)=-\frac{A^{\prime}(x)}{A(x)}$. Then system $(5.15)$ is equivalent to

$$
\left\{\begin{array}{l}
\rho_{t}+m_{x}=a(x) m \\
m_{t}+\left(\frac{m^{2}}{\rho}+p(\rho)\right)_{x}=a(x) \frac{m^{2}}{\rho}+G_{2}(\rho, m, x, t) .
\end{array}\right.
$$

As in Sect. 4, we construct the approximate solutions $\left(\rho^{h}(x, t), m^{h}(x, t)\right)$ of $(5.17)$ and then prove that the approximate solutions satisfy the compensated compactness framework (Lemma 5.5) as for (5.1). Then we conclude that there is a subsequence in the approximate solutions strongly converging to the $L^{\infty}$ function $(\rho(x, t), m(x, t))$ almost everywhere.

Theorem 5.4. Assume that the initial data $\left(\rho_{0}, u_{0}\right)$ are bounded in $L^{\infty}$. Then there exists a bounded weak entropy solution $(\rho(x, t), m(x, t))$ of $(5.15)-(5.16)$ in the sense of Definition 5.1.

\section{Two-Dimensional Symmetric Rotating Solutions}

Consider the solutions of (1.1) in $\mathbf{R}^{2}$ of the following form:

$$
(\rho, \vec{m}, \phi)(\vec{x}, t)=\left(\rho(|\vec{x}|, t), m(|\vec{x}|, t) \frac{\vec{x}}{|\vec{x}|}+\tilde{m}(|\vec{x}|, t) \frac{\left(-x_{2}, x_{1}\right)}{|\vec{x}|}, \phi(|\vec{x}|, t)\right),
$$

where $\vec{x}=\left(x_{1}, x_{2}\right)$. Then (1.1)-(1.2) becomes

$$
\left\{\begin{array}{l}
\rho_{t}+m_{x}=-\frac{1}{x} m \\
m_{t}+\left(\frac{m^{2}}{\rho}+p(\rho)\right)_{x}=-\frac{1}{x} \frac{m^{2}}{\rho}+G_{2}(\rho, m, \tilde{m}, x, t), \\
\tilde{m}_{t}+\left(\frac{m \tilde{m}}{\rho}\right)_{x}=-\frac{2}{x} \frac{m \tilde{m}}{\rho}-\frac{\tilde{m}}{\tau}
\end{array}\right.
$$

with the initial-boundary conditions:

$$
\left\{\begin{array}{l}
\left.(\rho, m, \tilde{m})\right|_{t=0}=\left(\rho_{0}(x), m_{0}(x), \tilde{m}_{0}(x)\right) \\
\left.m\right|_{x=1}=\left.m\right|_{x=2}=0
\end{array}\right.
$$

where $x=|\vec{x}|$ and $G_{2}=\rho x^{-1}\left(\int_{1}^{x}(\rho-D(\xi)) \xi d \xi+c(\rho, t)\right)+\frac{1}{x} \tilde{m}^{2} \rho-\frac{m}{\tau}$ with $c(\rho, t)$ the same as in (5.3) for $N=2$. 
Set $\hat{m}=\tilde{m} e^{\frac{t}{\tau}}, \hat{\omega}=\frac{\hat{m}}{\rho}=\omega e^{\frac{t}{\tau}}$. For any test function $\psi \in C_{0}^{1}\left(\Pi_{T}\right)$, taking $\hat{\psi}=\psi e^{-\frac{t}{\tau}} \in C_{0}^{1}\left(\Pi_{T}\right)$, one has

$$
\begin{gathered}
\iint_{\Pi_{T}}\left(\tilde{m} \psi_{t}+\frac{m \tilde{m}}{\rho} \psi_{x}+\left(-\frac{2}{x} \frac{m \tilde{m}}{\rho}-\frac{\tilde{m}}{\tau}\right) \psi\right) d x d t \\
\quad=\iint_{\Pi_{T}}\left(\hat{m} \hat{\psi}_{t}+\frac{m \hat{m}}{\rho} \hat{\psi}_{x}-\frac{2}{x} \frac{m \hat{m}}{\rho} \hat{\psi}\right) d x d t
\end{gathered}
$$

Then (6.1)-(6.2) is equivalent to

$$
\left\{\begin{array}{l}
v_{t}+f(v)_{x}=a(x) g(v)+G(v, x, t) \\
\left.v\right|_{t=0}=v_{0} \\
\left.\hat{m}\right|_{x=1}=\left.\hat{m}\right|_{x=2}=0
\end{array}\right.
$$

where $v=(\rho, m, \hat{m})^{\top} \equiv(\tilde{v}, \hat{m})^{\top}$ with $\tilde{v}=(\rho, m)^{\top}, a(x)=-\frac{1}{x}, f(v)=\left(m, \frac{m^{2}}{\rho}, \frac{m \hat{m}}{\rho}\right)^{\top}$, $g(v)=\left(m, \frac{m^{2}}{\rho}, \frac{2 m \hat{m}}{\rho}\right)^{\top}$, and $G=\left(0, \hat{G}_{2}, 0\right)^{\top}$ with $\hat{G}_{2}(\rho, m, \hat{m}, x, t)=G_{2}\left(\rho, m, \hat{m} e^{-\frac{t}{\tau}}, x, t\right)$.

We can construct the approximate solutions of (6.3) as the construction for (4.1) with $\bar{m}=(m, \hat{m})$. In this case, $(4.7)$ becomes

$$
\left\{\begin{array}{l}
\rho^{h}(x, t)=\rho_{0}^{h}(x, t), \quad \hat{m}^{h}(x, t)=\hat{m}_{0}^{h}(x, t), \\
m^{h}(x, t)=m_{0}^{h}(x, t)+\hat{G}_{2}\left(m_{0}^{h}(x, t), \hat{m}_{0}^{h}(x, t), x, t\right)(t-n \Delta t) .
\end{array}\right.
$$

Equivalently, $\left(\rho^{h}(x, t), m^{h}(x, t), \tilde{m}^{h}(x, t)\right)=\left(\rho^{h}(x, t), m^{h}(x, t), \hat{m}^{h}(x, t) e^{-\frac{t}{\tau}}\right)$ is the approximate solution of $(6.1)-(6.2)$.

In the strip $n \Delta t \leqq t<(n+1) \Delta t$, by the construction and Lemma 3.1, we obtain

$$
\left\{\begin{array}{l}
w\left(\tilde{v}_{0}^{h}(x, t)\right) \leqq \max \left(\sup _{x} w\left(\tilde{v}_{0}^{h}(x, n \Delta t+0)\right), 1\right)(1+C \Delta t), \\
z\left(\tilde{v}_{0}^{h}(x, t)\right) \geqq \min \left(\inf _{x} z\left(\tilde{v}_{0}^{h}(x, n \Delta t+0)\right),-1\right)(1+C \Delta t), \\
\left|\hat{\omega}_{0}^{h}(x, t)\right| \leqq \sup _{x}\left|\hat{\omega}_{0}^{h}(x, n \Delta t+0)\right|(1+\Delta t) .
\end{array}\right.
$$

Theorem 6.1. Assume that the initial data satisfy $\left|u_{0}(x)\right|+\left|\hat{\omega}_{0}(x)\right| \leqq C_{0}$, $0 \leqq \rho_{0}(x) \leqq C_{0}$, for some $C_{0}>0$. Then there exists a positive constant $\bar{C}(T)$, independent of $h$ and $\tau$, such that

$$
\left|u^{h}(x, t)\right|+\left|\hat{\omega}^{h}(x, t)\right| \leqq C(T), \quad h^{\beta} \leqq \rho^{h}(x, t) \leqq C(T), \quad(x, t) \in \Pi_{T} .
$$

Proof. For $\hat{\omega}^{h}(x, t)$, in the strip $n \Delta t \leqq t<(n+1) \Delta t$, from (6.4)

$$
\left|\hat{\omega}^{h}(x, t)\right|=\left|\hat{\omega}_{0}^{h}(x, t)\right| \leqq \sup _{x}\left|\hat{\omega}_{0}^{h}(x, n \Delta t+0)\right|(1+\Delta t) .
$$

Set $M_{n}=\sup _{x}\left|\hat{\omega}_{0}^{h}(x, n \Delta t+0)\right|$. Then $M_{n+1} \leqq M_{n}(1+\Delta t)$. Therefore

$$
M_{n+1} \leqq \sup _{x}\left|\hat{\omega}_{0}(x)\right|(1+\Delta t)^{n+1} \leqq C_{0}(1+\Delta t)^{\frac{T}{\Delta t}+1} \leqq C(T),
$$

which implies the uniform boundedness of $\hat{\omega}^{h}$. Then, by the same procedure of the proof of Theorem 5.1, we have the uniform estimate for $\rho^{h}$ and $u^{h}$. 
Following the arguments of Sect. 5.2, we can conclude

Theorem 6.2. Assume that $v^{h}=\left(\rho^{h}, m^{h}, \hat{m}^{h}\right) \equiv\left(\tilde{v}^{h}, \hat{m}^{h}\right)$ are the approximate solutions of (6.3). Then the measure sequence $\eta\left(\tilde{v}^{h}\right)_{t}+q\left(\tilde{v}^{h}\right)_{x}$ is a compact subset of $H_{\mathrm{loc}}^{-1}(\Omega)$ for all weak entropy pair $(\eta, q)$, where $\Omega \subset \Pi_{T}$ is any bounded and open set. results.

Then, similar to Sect.5.3, we have the following convergence and existence

Theorem 6.3. Assume that $v^{h}=\left(\rho^{h}, m^{h}, \hat{m}^{h}\right)$ are the approximate solutions of (6.3). Then there is a convergent subsequence (still labeled) $v^{h}$ such that

$$
v^{h}(x, t) \rightarrow v(x, t)=(\rho(x, t), m(x, t), \hat{m}(x, t)), \text { a.e. }
$$

and that the function $v(x, t)$ is a bounded weak entropy solution in the sense of Definition 5.1 .

Proof. The convergence of $\left(\rho^{h}(x, t), m^{h}(x, t)\right)$ follows from Theorem 6.1, Theorem 6.2, and Lemma 5.5. To show the convergence of $\hat{m}^{h}(x, t)$, we use the method of quasidecoupling (cf. [8]). By the similar argument in Sect. 5, we have

$$
\left(x \rho^{h} \bar{a}\left(x \hat{\omega}^{h}\right)\right)_{t}+\left(x m^{h} \bar{a}\left(x \hat{\omega}^{h}\right)\right)_{x} \leqq o(1) \rightarrow 0, \quad h \rightarrow 0,
$$

in the sense of distributions for any convex function $\bar{a} \in C^{2}$.

Define the probability measures $\mu_{x, t}$ by $\left\langle\mu_{x, t}, g(\hat{\omega})\right\rangle=\left\langle v_{x, t}, g(x \hat{\omega})\right\rangle$, where $v_{x, t}$ is the Young measure determined by the approximate solutions $v^{h}(x, t)$. Then $\mu_{x, t}$ satisfy

$$
\left(x \rho(x, t)\left\langle\mu_{x, t}, \bar{a}(\hat{\omega})\right\rangle\right)_{t}+\left(x m(x, t)\left\langle\mu_{x, t}, \bar{a}(\hat{\omega})\right\rangle\right)_{x} \leqq 0 .
$$

Note that $(x \rho(x, t))_{t}+(x m(x, t))_{x}=0$, in the generalized sense of distributions, $x \rho(x, t) \geqq 0$, and $\mu_{x, 0}=\delta_{\hat{\omega}_{0}(x)}$, since $\hat{\omega}_{0}^{h}(x) \rightarrow \hat{\omega}_{0}(x)$, a.e. By the refinement of Lemma 3.5 of [8] as in [10],

$$
\mu_{x, t}=\delta_{\hat{\omega}(x, t)}, \quad(x, t) \in \Pi_{T}-\{\rho(x, t)=0\} .
$$

This implies that $\hat{m}^{h}(x, t) \rightarrow \hat{m}(x, t)$, a.e. By the similar argument in Sect. 5, we can prove that $v(x, t)=(\rho(x, t), m(x, t), \hat{m}(x, t))$ is a weak entropy solution of $(6.3)$ and that, equivalently, $(\rho(x, t), m(x, t), \tilde{m}(x, t))=\left(\rho(x, t), m(x, t), \hat{m}(x, t) e^{-\frac{t}{\tau}}\right)$ is a weak entropy solution of $(6.1)-(6.2)$.

\section{Traces of Weak Solutions}

In this section, we consider the boundary values of the weak solutions. We first define the traces of the spherically symmetric solutions as in [28] and then prove that the weak solution satisfies the initial-boundary conditions, which implies that the initial-boundary conditions are well-posed. For the solutions with the other type of structure, the situation is the same.

Let $v=v(x, t),(x, t) \in(1,2) \times(0, T)$, be a weak solution of (5.1) satisfying (5.10). Define a function $\mathfrak{L}: C_{0}^{1}\left(\mathbf{R}^{2}\right) \rightarrow \mathbf{R}^{2}$ as:

$$
\mathfrak{L}(\psi)=-\iint_{\Pi_{T}}\left(v \psi_{t}+f(v) \psi_{x}+(a(x) g(v)+G) \psi\right) d x d t,
$$


for $\psi \in C_{0}^{1}\left(\mathbf{R}^{2}\right)$. Choose $\zeta_{0}, \zeta_{T}, \zeta_{1}, \zeta_{2} \in C_{0}^{1}(\mathbf{R})$ with

$$
\begin{aligned}
& \zeta_{0}(0)=1, \quad \zeta_{0}(T)=0 ; \quad \zeta_{T}(0)=0, \quad \zeta_{T}(T)=1 ; \\
& \zeta_{1}(1)=1, \quad \zeta_{1}(2)=0 ; \quad \zeta_{2}(1)=0, \quad \zeta_{2}(2)=1 \text {. }
\end{aligned}
$$

Then define the trace $v^{*}(\cdot, 0), v^{*}(\cdot, T): C_{0}^{1}(\mathbf{R}) \rightarrow \mathbf{R}^{2}$ of $v$ along the part $(1,2) \times$ $\{0\},(1,2) \times\{T\}$ of the boundary of $\Pi_{T}$, and the trace $f^{*}(v)(1, \cdot), f^{*}(v)(2, \cdot)$ : $C_{0}^{1}(\mathbf{R}) \rightarrow \mathbf{R}^{2}$ of $f(v)$ along the part $\{1\} \times(0, T),\{2\} \times(0, T)$ of the boundary of $\Pi_{T}$ as:

$$
\begin{aligned}
& v^{*}(\cdot, 0)(\chi)=\mathfrak{L}\left(\chi \cdot \zeta_{0}\right)-\chi(1) \mathfrak{L}\left(\zeta_{1} \cdot \zeta_{0}\right)-\chi(2) \mathfrak{L}\left(\zeta_{2} \cdot \zeta_{0}\right), \\
& v^{*}(\cdot, T)(\chi)=-\mathfrak{L}\left(\chi \cdot \zeta_{T}\right)+\chi(1) \mathfrak{L}\left(\zeta_{1} \cdot \zeta_{T}\right)+\chi(2) \mathfrak{L}\left(\zeta_{2} \cdot \zeta_{T}\right), \\
& f^{*}(v)(1, \cdot)(\chi)=\mathfrak{L}\left(\zeta_{1} \cdot \chi\right), \quad f^{*}(v)(2, \cdot)(\chi)=-\mathfrak{L}\left(\zeta_{2} \cdot \chi\right),
\end{aligned}
$$

for $\chi \in C_{0}^{1}(\mathbf{R})$, where $\chi \cdot \zeta_{0}(x, t)=\chi(x) \zeta_{0}(t)$, etc. For any $t \in(0, T)$, we can also define $v^{*}(\cdot, t)$ as the trace of $v$ along the part $(1,2) \times\{t\}$ of the boundary of $(1,2) \times(0, t)$ or $(1,2) \times(t, T)$. For any $x \in(1,2)$, define $f^{*}(v)(x, \cdot)$ as the trace of $f(v)$ along the part $\{x\} \times(0, T)$ of the boundary of $(1, x) \times(0, T)$, or $(x, 2) \times(0, T)$. Then, as in [28], we have the following lemma.

Lemma 7.1. Let $v$ be a weak solution of (5.1) satisfying (5.10). Then

(1) $\left.v^{*}(\cdot, 0)\right|_{(1,2)},\left.v^{*}(\cdot, T)\right|_{(1,2)} \in L_{\mathrm{loc}}^{\infty}(1,2) ;\left.f^{*}(v)(1, \cdot)\right|_{(0, T)},\left.f^{*}(v)(2, \cdot)\right|_{(0, T)} \in$ $L_{\mathrm{loc}}^{\infty}(0, T)$, and for any $\psi \in C_{0}^{1}\left(\mathbf{R}^{2}\right)$,

$$
\begin{aligned}
\iint_{\Pi_{T}}\left(v \psi_{t}+f(v) \psi_{x}+(a(x) g(v)+G(v, x, t)) \psi\right) d x d t \\
=\int_{1}^{2} v^{*}(x, T) \psi(x, T) d x-\int_{1}^{2} v^{*}(x, 0) \psi(x, 0) d x \\
\quad+\int_{0}^{T} f^{*}(v)(2, t) \psi(2, t) d t-\int_{0}^{T} f^{*}(v)(1, t) \psi(1, t) d t .
\end{aligned}
$$

(2) For all $x_{1}<x_{2}, t_{1}<t_{2}$, with $\left(x_{1}, x_{2}\right) \times\left(t_{1}, t_{2}\right) \subset \Pi_{T}$, the functions

$$
\left[t_{1}, t_{2}\right] \rightarrow \mathbf{R}^{2}, t \mapsto \int_{x_{1}}^{x_{2}} v^{*}(x, t) d x ; \quad\left[x_{1}, x_{2}\right] \rightarrow \mathbf{R}^{2}, x \mapsto \int_{t_{1}}^{t_{2}} f^{*}(v)(x, t) d t
$$

are uniformly Lipschitz continuous.

Now we prove that the traces satisfy the initial-boundary conditions.

Theorem 7.1. Let $v^{h}(x, t)=\left(\rho^{h}(x, t), m^{h}(x, t)\right)$ be the approximate solutions of (5.1) constructed in Sect. 4 and $v(x, t)=(\rho(x, t), m(x, t))$ is the limit function of $v^{h}$ as $h \rightarrow 0$. Then $v(x, t)$ satisfies the initial-boundary conditions:

$$
\left\{\begin{array}{l}
v^{*}(x, 0)=v_{0}(x), x \in(1,2) \\
m^{*}(1, t)=m^{*}(2, t)=0, \quad t \in(0, T) .
\end{array}\right.
$$


Proof. By the construction of $v^{h}$, for any $\psi \in C_{0}^{1}\left(\mathbf{R}^{2}\right), \bar{\psi}(x, t)=\frac{\psi(x, t)}{A(x)} \in C_{0}^{1}\left(\mathbf{R}^{2}\right)$, one has

$$
\begin{aligned}
& \iint_{\Pi_{T}}\left(\rho^{h} \psi_{t}+m^{h} \psi_{x}+a(x) m^{h} \psi\right) d x d t+\int_{1}^{2} \rho^{h}(x, 0) \psi(x, 0) d x-\int_{1}^{2} \rho^{h}(x, T) \psi(x, T) d x \\
& =\int_{\Pi_{T}}\left(A(x) \rho^{h} \bar{\psi}_{t}+A(x) m^{h} \bar{\psi}_{x}\right) d x d t+\int_{1}^{2} A(x) \rho^{h}(x, 0) \bar{\psi}(x, 0) d x \\
& \quad-\int_{1}^{2} A(x) \rho^{h}(x, T) \bar{\psi}(x, T) d x \\
& =\int_{\Pi_{T}} A(x)\left(m^{h}-m_{0}^{h}\right) \bar{\psi}_{x} d x d t+\int_{0}^{T} \sum A(x(t))\left(\sigma\left[\rho_{0}^{h}\right]-\left[m_{0}^{h}\right]\right) \bar{\psi}(x(t), t) d t \\
& \quad+\sum_{j, n} \int_{1+\left(j-\frac{1}{2}\right) h}^{1+\left(j+\frac{1}{2}\right) h} A(x)\left(\rho^{h}(x, n \Delta t-0)-\rho^{h}(x, n \Delta t+0)\right) \bar{\psi}(x, n \Delta t) d x+C h^{\beta}\|\bar{\psi}\|_{H^{1}} .
\end{aligned}
$$

Note that, by the fractional step procedure and the Rankine-Hugoniot condition,

$$
\begin{gathered}
\iint_{\Pi_{T}} A(x)\left(m^{h}-m_{0}^{h}\right) \bar{\psi}_{x} d x d t=O(h), \\
\int_{0}^{T} \sum A(x(t))\left(\sigma\left[\rho_{0}^{h}\right]-\left[m_{0}^{h}\right]\right) \bar{\psi}(x(t), t) d t=0,
\end{gathered}
$$

and by the similar estimate in Sect. 5.3,

$$
\sum_{j, n} \int_{1+\left(j-\frac{1}{2}\right) h}^{1+\left(j+\frac{1}{2}\right) h} A(x)\left(\rho^{h}(x, n \Delta t-0)-\rho^{h}(x, n \Delta t+0)\right) \bar{\psi}(x, n \Delta t) d x=O\left(h^{\beta}\right) .
$$

Taking the limit $h \rightarrow 0$ in (7.2) and using the above estimate, one has $\iint_{\Pi_{T}}\left(\rho \psi_{t}+m \psi_{x}+a(x) m \psi\right) d x d t+\int_{1}^{2} \rho_{0}(x) \psi(x, 0) d x-\lim _{h \rightarrow 0} \int_{1}^{2} \rho^{h}(x, T) \psi(x, T) d x=0$.

Using (7.1) to substitute the first integral of the above equality, we have

$$
\begin{aligned}
& \int_{0}^{T} m^{*}(2, t) \psi(2, t) d t-\int_{0}^{T} m^{*}(1, t) \psi(1, t) d t+\int_{1}^{2} \rho_{0}(x) \psi(x, 0) d x-\int_{1}^{2} \rho^{*}(x, 0) \psi(x, 0) d x \\
& \quad+\int_{1}^{2} \rho^{*}(x, T) \psi(x, T) d x-\lim _{h \rightarrow 0} \int_{1}^{2} \rho^{h}(x, T) \psi(x, T) d x=0 .
\end{aligned}
$$

Take $\psi(x, t)=\zeta(x) \chi(t) \in C_{0}^{1}\left(\mathbf{R}^{2}\right)$ with $\zeta, \chi \in C_{0}^{1}(\mathbf{R})$, and $\chi(0)=1, \chi(T)=0$, $\zeta(1)=\zeta(2)=0$. One has

$$
\int_{1}^{2} \rho^{*}(x, 0) \zeta(x) d x=\int_{1}^{2} \rho_{0}(x) \zeta(x) d x,
$$


which implies $\rho^{*}(x, 0)=\rho_{0}(x)$ on $(1,2)$. Similarly, one has $m^{*}(x, 0)=m_{0}(x)$ on $(1,2)$.

Take $\psi(x, t)=\zeta(x) \chi(t) \in C_{0}^{1}\left(\mathbf{R}^{2}\right)$ in (7.3) with $\zeta, \chi \in C_{0}^{1}(\mathbf{R})$, and $\chi(T)=$ $\chi(0)=0, \zeta(1)=1, \zeta(2)=0$. Then one has

$$
\int_{0}^{T} m^{*}(1, t) \chi(t) d t=0 .
$$

Therefore $m^{*}(1, t)=0$ on $(0, T)$. Similarly, one concludes $m^{*}(2, t)=0$ on $(0, T)$.

Acknowledgements. The authors would like to thank Professor Joseph Jerome for stimulating conversations. Gui-Qiang Chen was supported in part by the Office of Naval Research grant N00014-91-J-1384, the National Science Foundation grants DMS-9623203, and an Alfred P Sloan Foundation Fellowship

\section{References}

1 Ascher, U.M, Markowich, P.A, Pietra, P., Schmeiser, C : A phase plane analysis of transonic solutions for the hydrodynamic semiconductor model. Math. Mod. Meth Appl. Sci. 1(3), 347-376 (1991)

2 Baccarani, G, Wordeman, M R : An investigation of steady-state velocity overshoot effects in $\mathrm{Si}$ and GaAs devices Solid State Electron 28, 404-416 (1985)

3 Bell, T E : The quest for ballistic action. IEEE Spectrum 23, 36-38 (1986)

4. Blotekjaer, K: Transport equations for electrons in two-valley semiconductors IEEE Transactions on Electron Devices, ED-17, 1, 38-47 (1970)

5 Chen, D P, Eisenberg, R.S., Jerome, J.W., Shu, C W : A hydrodynamic model of temperature change in open ionic channels Biophys J 69, 2304-2322 (1995)

6. Chen, G.-Q : Convergence of Lax-Friedrichs scheme for isentropic gas dynamics, III. Acta Math Sci 8, 243-276 (1988) (in Chinese); 6, 75-120 (1986)

7 Chen, G.-Q: The compensated compactness method and the system of isentropic gas dynamics MSRI Preprint 00527-91, Berkeley (1990)

8 Chen, G -Q: The method of quasidecoupling to conservation laws Arch Rat. Mech Anal 121, 131-185 (1992)

9 Chen, G-Q, Glimm, J.: Global solutions to the compressible Euler equations with geometrical structure. Commun. Math. Phys. (to appear) (1996)

10 Chen, G-Q., Glimm, J.: Global solutions to the cylindrically symmetric rotating motion of isentropic gases Z. angew. Math Phys 47 (1996) (to appear)

11 Chen, Z, Cockburn, B., Gardner, C.L, Jerome, J: Quantum hydrodynamic simulation of hysteresis in the resonant tunneling diode. J Comp. Phys. 117, 274-280 (1995)

12 Cordier, S, Degond, P., Markowich, P., Schmeiser, C: Traveling wave analysis and jump relations for Euler-Poisson model in the quasineutral limit, Preprint (1994)

13. Dafermos, C: Polygonal approximations of solutions of the initial-value problem for a conservation law J Math. Anal Appl. 38, 33-41 (1972)

14 Degond, P, Markowich, P A : On a one-dimensional steady-state hydrodynamic model for semiconductors Appl. Math Letters 3, 25-29 (1990)

15 Ding, X., Chen, G.-Q., Luo, P.: Convergence of the Lax-Friedrichs scheme for isentropic gas dynamics (I)-(II). Acta Mathematica Scientia 7, 467-480 (1987); 8, 61-94 (1988) (in Chinese); 5, 415-432, 433-472 (1985)

16. Ding, X., Chen, G.-Q., Luo, P.: Convergence of the fractional step Lax-Friedrichs scheme and Godunov scheme for the isentropic system of gas dynamics. Commun. Math. Phys. 121, 63-84 (1989)

17 DiPerna, R.: Convergence of the viscosity method for isentropic gas dynamics. Commun. Math. Phys. 91, 1-30 (1983)

18 Embid, P, Goodman, J., Majda, A : Multiple steady state for 1-D transonic flow. SIAM J. Sci Stat Comp 5, 21-41 (1984)

19 Fang, W., Ito, K.: One-dimensional hydrodynamic model for semiconductors by viscosity method Proc International Conf. on Diff Eqs, Claremont, California, June 1994, to appear 
20. Fatemi, E., Jerome, J., Osher, S.: Solution of the hydrodynamic device model using highorder nonoscillatory shock capturing algorithms. IEEE Transactions on Computer-Aided Design 10. 2, 232-243 (1991)

21. Gamba, I.M.: Stationary transonic solutions of a one-dimensional hydrodynamic model for semiconductors. Comm. P.D.E. 17, 553-577 (1992)

22. Gamba, I M.: Viscosity approximating solutions to ODE systems that admit shocks and their limits: Preprint (1994)

23. Gamba, I.M., Morawetz, C.: A viscous approximation for a 2D steady semiconductor or transonic gas dynamic flow: Existence theorem for potential flow Preprint, Courant Institute (1995)

24. Gardner, C L : Numerical simulation of a steady-state electron shock wave in a submicron semiconductor device. IEEE Transactions on Electron Devices 38, 392-398 (1991)

25. Gilbarg, D, Trudinger, N.S.: Elliptic Partial Differential Equations of Second Order. Berlin, Heidelberg, New York: Springer 1983

26. Glaz, H., Liu, T.-P.: The asymptotic analysis of wave interactions and numerical calculation of transonic nozzle flow. Avd. Appl. Math. 5, 111-146 (1984)

27. Glimm, J., Marshall, G., Plohr, B.: A generalized Riemann problem for quasi-one-dimensional gas flow. Adv. Appl. Math. 5, 1-30 (1984)

28. Heidrich, A : Global weak solutions to initial boundary value problems for the wave equation with large data. Arch. Rat. Mech. Anal. 126, 333-368 (1994)

29. Jochmann, F.: Global weak solutions of the one-dimensional hydrodynamic model for semiconductors. Math. Mod. Meth Appl. Sci. 3, 759-788 (1993)

30. Lax, P.D.: Hyperbolic Systems of Conservation Laws and the Mathematical Theory of Shock Waves. CBMS. 11, SIAM 1973

31 LeFloch, P.G: Glimm method applied to quasilinear hyperbolic systems with source-term Preprint 218, Ecole Polytechnique, France, 1990

32. Lions, P.L., Perthame, B., Tadmor, E.: Kinetic formulation of the isentropic gas dynamics and p-systems. Commun. Math. Phys. 163, 415-431 (1993)

33. Liu, T.-P.: Quasilinear hyperbolic system. Commun. Math. Phys. 68, 141-172 (1979)

34. Liu, T.-P.: Nonlinear resonance for quasilinear hyperbolic equation. J. Math. Phys. 28, 2593-2602 (1987)

35. Makino, T., Takeno, S.: Initial boundary value problem for the spherically symmetric motion of isentropic gas. Japan J. Indust. Appl Math 11, 171-183 (1994)

36. Marcati, P., Natalini, R.: Weak solutions to a hydrodynamic model for semiconductors and relaxation to the drift-diffusion equation. Arch. Rat. Mech. Anal. 129, 129-145 (1995)

37. Markowich, P.A., Ringhofer, C., Schmeiser, C.: Semiconductor Equations Berlin, Heidelberg, New York: Springer 1990

38. Poupaud, F., Rascle, M, Vila, J.P.: Global solutions to the Isothermal Euler-Poisson system with arbitrary large data. J. Diff. Eqs. 123, 93-121 (1995)

39. Roe, P.L.: Upwind differencing schemes for hyperbolic conservation laws with source terms. Lecture Notes in Math., Nonlinear Hyperbolic Problems, vol. 1270, Berlin, Heidelberg, New York: Springer 1986, pp. 41-51

40. Van Leer, B.: On the relation between the upwind-differencing scheme of Godunov, EngquistOsher and Roe. SIAM J. Sci. Stat. Comp. 5, 1-19 (1984)

41. Van Roosbroeck, W.V.: Theory of flow of electrons and holes in germanium and other semiconductors. Bell Syst. Techn. J. 29, 560-607 (1950)

42. Smoller, J.: Shock Waves and Reaction-Diffusion Equations. Berlin, Heidelberg, New York: Springer 1994

43. Tartar, L.: Compensated compactness and applications to partial differential equations. Research Notes in Mathematics, Nonlinear Analysis and Mechanics R.J. Knops (ed.) vol. 4, New York: Pitman Press 4, 1979

44. Zhang, B.: Convergence of the Godunov scheme for a simplified one-dimensional hydrodynamic model for semiconductor devices Commun. Math. Phys. 157, 1-22 (1993)

45. Zhang, B., Jerome, J.: On a steady-state quantum hydrodynamic model for semiconductors. Nonlinear Analysis 26, 845-856 (1996) 